\title{
Exciton spectra in vertical stacks of triple and quadruple quantum dots in an electric field
}

\author{
B. Szafran, ${ }^{1}$ E. Barczyk, ${ }^{1}$ F. M. Peeters, ${ }^{2}$ and S. Bednarek ${ }^{1}$ \\ ${ }^{1}$ Faculty of Physics and Applied Computer Science, AGH University of Science and Technology, \\ al. Mickiewicza 30, 30-059 Kraków, Poland \\ ${ }^{2}$ Departement Fysica, Universiteit Antwerpen, Groenenborgerlaan 171, B-2020 Antwerpen, Belgium \\ (Received 10 September 2007; revised manuscript received 20 December 2007; published 24 March 2008)

\begin{abstract}
We study an electron-hole pair in a stack of multiple quantum dots in the presence of an external electric field using the configuration-interaction approach. We find that the bright energy levels can be grouped into families which are associated with the hole localized in a specific dot of the stack. The exciton energy levels undergo avoided crossings as function of the external electric field with different patterns for each family. We show that the variation of the depths of the dots along the stack can be deduced from the exciton spectrum. For stronger coupling the families are mixed by a weak electric field due to hole tunneling. This results in a characteristic multiple avoided crossing of energy levels belonging to different families with an accompanying modulation of the recombination probabilities and an appearance of a single particularly bright state. Discussion of a simple modeling of these avoided crossings is provided.
\end{abstract}

DOI: $10.1103 /$ PhysRevB.77.115441

PACS number(s): 73.21.La, 73.43.-f, 71.10.Pm

\section{INTRODUCTION}

Double quantum dots are considered promising for construction of basic quantum information processing devices with electron spins ${ }^{1}$ or excitons ${ }^{2}$ confined in separate dots acting as quantum bits interacting in a way that can be controlled, for instance, by an external electric field. One technique used for double dot formation uses the spontaneous tendency of the self-assembled InGaAs quantum dots to stack one above the other during epitaxial growth. ${ }^{3-6}$ Coupling between the dots within a stack was detected early in photoluminescence experiments ${ }^{3,4,7}$ as redshifts of the exciton energy lines that appear when the interdot barrier thickness is reduced. Redshifts are also observed as function of the number of dots forming the stack. ${ }^{3,4}$ Only relatively recently measurements of the photoluminescence spectrum with an external electric field applied to a pair of dots were performed. ${ }^{8-15}$ These measurements ${ }^{8-15}$ exploit the optical signatures of coupling between the dots to establish the role of electron and hole tunneling as well as the interaction between the carriers in the artificial molecules. They proved useful for probing the confinement potential in asymmetric quantum dots,${ }^{11}$ examining the spin interactions in the fine structure of the photoluminescence lines ${ }^{15}$ and the observation of an electric-field tunable $g$ factor. ${ }^{14}$

In quantum information processing the idea of using a pair of quantum dots as coupled quantum bits implies as a natural extension an array of quantum dots as a scalable quantum register. ${ }^{16}$ Recently, there has been a growing interest in artificial molecules formed by more than two dots. For instance, multiple coupled dots ${ }^{17}$ were used for construction of a high accuracy single-electron pump. It was demonstrated ${ }^{18}$ that a triple quantum dot can be used to construct gates for multivalued logic. Moreover, usage of three quantum dots as a source of spin-entangled currents was demonstrated. ${ }^{19}$ Stability diagram of an artificial molecule formed by three planar dots created in a gated twodimensional electron gas was determined. ${ }^{20}$ Artificial molecules formed of multiple self-assembled quantum dots in planar geometry were also fabricated. ${ }^{21-23}$
Till now theoretical ${ }^{24-28}$ and experimental work ${ }^{8-15}$ on the electric field effect on photoluminescence spectra was limited to artificial molecules formed by pairs of dots. The purpose of the present paper is to extend the previous theoretical work on the exciton spectrum of two vertically coupled quantum dots to a vertical stack of three and four quantum dots. The studied objects are an intermediate stage between the thoroughly studied case of single and double dots and the dot superlattice that is eventually formed with a large number of stacked dots. We discuss the evolution of the spectra and electron-hole correlations when subsequent dots are added to the stack. According to the results presented below the spectra contain spectacular anomalous multiple avoided crossings of bright energy levels related to both the electron and hole tunneling which may be readily experimentally verified since both sample growth and measurement techniques are available.

The electric field breaks the electron tunnel coupling between the dots and separates electrons from holes. For a pair of dots the dissociation of the exciton by the removal of the electron from the deeper dot leads to a characteristic avoided crossing of a bright energy level and a dark one ${ }^{8,9}$ with spatially separated carriers. Most of the experiments ${ }^{8-13}$ were performed in the intermediate-coupling regime with barrier thickness that allows only the electron tunneling, with a hole localized in a single dot. In such a case the optical signatures of the interdot coupling are determined completely by the electron $\operatorname{transfer}^{8-13}$ induced by the electric field. However, avoided crossings related to tunneling of the hole are also observed $^{14}$ in the strong-coupling limit. In the present paper we discuss both the intermediate and strong coupling between the dots. We find that in the intermediate-coupling case the apparently complex spectra are ordered by the hole which stays localized in a specific dot within the stack. The position of the hole-containing dot in the stack leads to an individual pattern of avoided crossings when the extended electron states are manipulated by the electric field. This can be used to probe the variation of the confinement potential along the stack by means of photoluminescence measurements as previously applied for intentionally grown asym- 
metric double quantum dots. ${ }^{11}$ In the strong-coupling limit we obtain avoided crossings that are due to the hole tunneling, with a strong modulation of the recombination probabilities favoring a single energy level in the center of the crossing. Outside the avoided crossing range the hole becomes localized in a single dot and the spectra evolve with the electric field like in the intermediate-coupling case.

Although the physics of the interdot coupling can be most conveniently described by a model of identical dots, the dots that are actually produced are never identical. ${ }^{3,4}$ For a pair of dots one of them is always larger and/or deeper than the other. For more than two dots a number of possibilities in the variation of the depth of confinement potential along the stack are possible. The purpose of the present paper is to extract the features of the spectra that are independent of a specific order of confinement potential depths along the stack. For that reason we consider both systems of identical dots as well as dots with varied potential depths. Of the latter we mainly exploit the realistic case in which the depth of the confinement potential grows along the stack (below, this case is referred to as a "constant gradient"). This is motivated by the fact that in the Stranski-Krastanov growth mode for the same nominal number of InAs monolayers deposited for each layer of dots, the size of the quantum dots tends to increase along the stack. ${ }^{3,4}$ However, since the potential of separate dots can be intentionally modified ${ }^{11}$ during the growth also other configurations will be considered in this paper, namely, those in which the dot with the strongest confinement is situated inside the stack and not on its end.

We apply our configuration-interaction approach introduced in Ref. 24 and developed further in Ref. 27 which successfully predicted ${ }^{24}$ the mechanism of the dissociation of the exciton ${ }^{8,9}$ and the trion ${ }^{12}$ by the electric field.

The paper is organized as follows. In Sec. II we describe the model. Section III contains the results for an ideal system of three identical dots which are then generalized to the case of three and four nonidentical dots. Results are discussed in Sec. IV. Conclusions and summary are given in Sec. V.

\section{MODEL AND METHOD}

The model and the computational method are those as were presented in Ref. 27 for two coupled dots. This approach accounts for the electron-hole correlation in the external electric field assuming the single valence band approximation and a simple confinement potential model. The single band approximation for the hole is justified by a small height of the dot which excludes the light hole contribution from the lowest-energy states. The dipole moment induced by the charge transfer between the dots is much larger than the one resulting from the wave function deformation within a single dot. Therefore, we choose to apply a simple confinement potential model. For a single dot we use a disk quantum well,

$$
V(\mathbf{r})=-V_{0} /\left\{\left(1+\frac{x^{2}+y^{2}}{R^{2}}\right)^{10}\left[1+\frac{(z-\zeta)^{2}}{Z^{2}}\right]^{10}\right\},
$$

where $\zeta$ is the position of the center of the dot along the $z$ axis. The profile of the confinement potential is plotted by

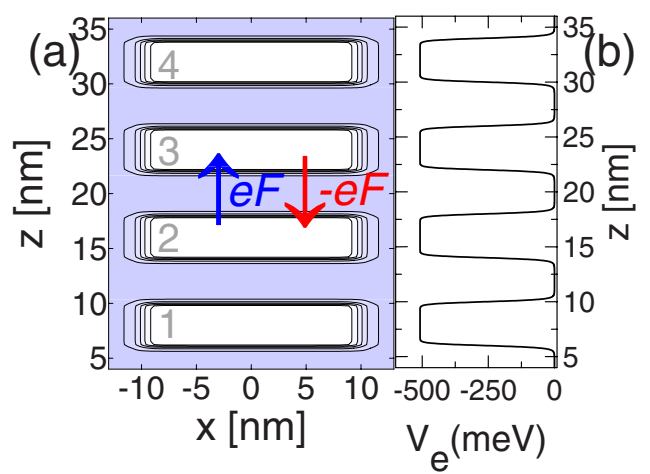

FIG. 1. (Color online) (a) The confinement potential (cross section at $y=0)$ for a stack of dots with interdot barrier of $4 \mathrm{~nm}$-the lighter the shade of gray, the deeper the potential. Dots have identical size with diameter of $20 \mathrm{~nm}$ and height of $4 \mathrm{~nm}$. The dots are numbered from the lowest to the uppermost. The arrow at left (right) shows the electric field force acting on the electron (hole) for $F>0$. (b) The confinement potential for the electron plotted along the axis of the stack $(x=0, y=0)$.

shades of gray in Fig. 1(a) for a stack of four identical dots and Fig. 1(b) presents the electron confinement potential along the axis of the stack.

The dots are assumed to be perfectly aligned [see Fig. 1(a)]. A nonperfect alignment has a negligible effect on the spectra when the electric field is oriented in the growth direction. ${ }^{27}$ Following the experimental data of Ref. 12 we take the diameter of the dots $2 R$ equal to $20 \mathrm{~nm}$ and the height $2 Z$ to $4 \mathrm{~nm}$. The effective masses $\left(m_{e}=0.037 m_{0}\right.$ for the electron and $m_{h}=0.45 m_{0}$ for the hole) as well as the dielectric constant $(\epsilon=12.5)$ are taken for $\operatorname{In}_{0.66} \mathrm{Ga}_{0.34} \mathrm{As}$ quantum $\operatorname{dot}^{27}$ embedded in GaAs and consequently the depth of the wells for the electron and hole are, respectively, $V_{0}^{e}=508 \mathrm{meV}$ and $V_{0}^{h}=218 \mathrm{meV}$. A variation of the confinement potential within the stack is modeled by introducing variations in the depths of the dots. We consider the following Hamiltonian for an electron-hole pair:

$$
\begin{aligned}
H= & -\frac{\hbar^{2}}{2 m_{e}} \nabla_{e}^{2}-\frac{\hbar^{2}}{2 m_{h}} \nabla_{h}^{2}+V_{e}\left(\mathbf{r}_{e}\right)+V_{h}\left(\mathbf{r}_{h}\right) \\
& -\frac{e^{2}}{4 \pi \epsilon \epsilon_{0}\left|\mathbf{r}_{e}-\mathbf{r}_{h}\right|}-e \mathbf{F} \cdot\left(\mathbf{r}_{e}-\mathbf{r}_{h}\right),
\end{aligned}
$$

where $\mathbf{r}_{e}, \mathbf{r}_{h}$ are the electron and the hole coordinates, respectively, the confinement potentials for the electron and hole are given by a sum of single-dot potentials of the form given by Eq. (1), and $\mathbf{F}$ is the electric field. In this Hamiltonian the energy is calculated with respect to the bottom of the conduction and the top of the valence band of the barrier material (GaAs). The electron-hole exchange interaction is small $^{15}$ at the energy scale of the avoided crossings and is therefore neglected in the present paper.

The electron-hole pair eigenproblem is solved using the configuration-interaction method with the single-particle eigenfunctions $\left[f_{p}^{(j)}\left(\mathbf{r}_{p}\right)\right.$ where $j$ enumerates the singleparticle eigenfunctions and the particle notation is $p=e$ for 
the electron and $p=h$ for the hole] diagonalized in the multicenter basis of Gaussian wave functions ${ }^{27}$

$$
\begin{aligned}
f_{p}^{(j)}\left(\mathbf{r}_{p}\right)= & \sum_{i} c_{i}^{(j, p)} \exp \left\{-\alpha_{i}^{p}\left[\left(x_{p}-x_{i}^{p}\right)^{2}\right.\right. \\
& \left.\left.+\left(y_{p}-y_{i}^{p}\right)^{2}\right]-\beta_{i}^{p}\left(z_{p}-z_{i}^{p}\right)^{2}\right\},
\end{aligned}
$$

where $c_{i}^{(j, p)}$ are the linear variational parameters and $\alpha_{i}^{p}$ and $\beta_{i}^{p}$ are the nonlinear variational parameters describing the localization strength of $i$ th Gaussian around point $\left(x_{i}^{p}, y_{i}^{p}, z_{i}^{p}\right)$. For each dot we apply 11 Gaussian functions: 8 Gaussians on a circle around the axis of the stack and 3 Gaussians along the axis. Exact positions of the Gaussians and strength of their localization in the growth direction $\left(\beta_{i}^{p}\right)$ and in the perpendicular plane $\left(\alpha_{i}^{p}\right)$ are optimized variationally, separately for the electron and for the hole. The basis accounts for confined single-particle states with angular momentum up to $\pm 4 \hbar$. Inclusion of more Gaussian centers in the basis does not significantly improve the results. In total, for a stack of $N$ dots the basis for the electron-hole pair contains $(11 N)^{2}$ localized wave functions given by products of single-electron $f_{e}^{(j)}$ and single-hole functions $f_{h}^{(k)}$

$$
\phi\left(\mathbf{r}_{e}, \mathbf{r}_{h}\right)=\sum_{j=1}^{11 N} \sum_{k=1}^{11 N} d_{j k} f_{e}^{(j)}\left(\mathbf{r}_{e}\right) f_{h}^{(k)}\left(\mathbf{r}_{h}\right) .
$$

We discuss the electron $n_{e}\left(\mathbf{r}_{e}\right)$ and hole $n_{h}\left(\mathbf{r}_{h}\right)$ densities which are extracted from the exciton wave function $\phi$ whose arguments are vectors in six-dimensional space by integrating its square over the coordinates of the other particle, i.e.,

$$
n_{e}\left(\mathbf{r}_{e}\right)=\int d \mathbf{r}_{h}\left|\phi\left(\mathbf{r}_{e}, \mathbf{r}_{h}\right)\right|^{2}
$$

and

$$
n_{h}\left(\mathbf{r}_{h}\right)=\int d \mathbf{r}_{e}\left|\phi\left(\mathbf{r}_{e}, \mathbf{r}_{h}\right)\right|^{2} .
$$

Finally, the recombination probability for the exciton state wave function $\phi$ is calculated as

$$
p=\left|\int d^{3} \mathbf{r}_{e} \int d^{3} \mathbf{r}_{h} \phi\left(\mathbf{r}_{e}, \mathbf{r}_{h}\right) \delta^{3}\left(\mathbf{r}_{e}-\mathbf{r}_{h}\right)\right|^{2} .
$$

At the end of this section we would like to point out that the present model is applied to a single stack of tunnelcoupled dots. Quantum dots grown in arrays may interact with one another through the electromagnetic field even though there is no tunneling between the dots. Electromagnetic coupling effects include stimulated emission amplification, ${ }^{29,30}$ polarization splitting of the gain band ${ }^{31}$ observed at high excitation intensity in the arrays of vertically stacked dots, and super-radiance observed ${ }^{32}$ for spontaneous emission at weak excitations. Experimentally, separation of a single stack of dots can be performed, for instance, by etching a small mesa of the entire sample. ${ }^{32}$

\section{RESULTS}

In the present paper we discuss a stack of intermediately and strongly coupled dots (see Sec. I). The strength of the coupling can be conveniently quantified by the energy splitting of binding and antibinding states for a pair of identical dots. For intermediate coupling the electron splitting is of order $10 \mathrm{meV}$. The hole tunneling remains negligible, with the energy splitting smaller than $100 \mu \mathrm{eV}$. The results presented below for the intermediate-coupling regime were obtained for barrier thickness $t$ between 4 and $6 \mathrm{~nm}$. For a pair of identical dots we obtain the electron splittings of 9.5 and $27.5 \mathrm{meV}$ for $t=6$ and $4 \mathrm{~nm}$, respectively. Corresponding hole energy splittings at these values of $t$ are 4 and $70 \mu \mathrm{eV}$. As a strong-coupling case we discuss spectra obtained for $t$ $=1 \mathrm{~nm}$ thick barrier. For $t=1 \mathrm{~nm}$ the electron splitting is as large as $136 \mathrm{meV}$, which largely exceeds a possible unintentional variation of the confinement depths between the dots. This is not the case for the hole, for which the tunnel coupling becomes significant but the energy splitting equals only $5.8 \mathrm{meV}$. For the applied potential parameters the groundstate energy of the exciton in a single quantum dot equals $-460.7 \mathrm{meV}$. The ground state becomes redshifted with the addition of subsequent identical dots to the stack: for interdot barrier of $1 \mathrm{~nm}$ we obtain ground-state energies of -524.4 , -546.8 , and $-556.2 \mathrm{meV}$ for the stack of two, three, and four dots, respectively. The corresponding relative shifts with addition of second, third, and fourth dots are equal to 63.8, 22.4 , and $9.4 \mathrm{meV}$. With the addition of subsequent dots the spectrum tends toward the superlattice limit. This is the reason why the redshift reduces with addition of dots to the stack. The redshift of the ground-state energy as well as the obtained reduction of the relative shifts with the number of dots is in qualitative agreement with the experimental results given for a sample containing strongly coupled stacks of several dots (see Fig. 4 of Ref. 4). However, in the experimental work ${ }^{4}$ the corresponding relative shifts are 64.1,34.3, and $32.8 \mathrm{meV}$, i.e., they reduce with the number of dots in a less pronounced manner than in our results for identical dots. This may be, for instance, due to the fact that in this experiment ${ }^{4}$ the size of the dots grows as they are added on top of the stack.

For our discussion of the evolution of the photoluminescence spectrum with increasing number of dots within the stack it is useful to inspect the spectrum for a couple of identical dots. This is given in Fig. 2 for intermediate coupling $(t=5 \mathrm{~nm})$ as function of the electric field $F$. The spectrum contains a set of bright energy levels [the thickness of the curves is set proportional to the recombination probability calculated according to Eq. (7)] and a multitude of dark energy levels corresponding to hole excitations with nonzero angular momenta and/or separated carriers. For intermediate coupling the bright levels can be divided into families which differ by the dot in which the hole is localized. The families are labeled by numbers that indicate the hole-containing dot in accordance with the order indicated schematically in Fig. 1(a). Within each family we additionally label the energy levels that appear as bright when the electric field is swept from strong negative values to strong positive values by subsequent letters $a, b, c$, etc.

Energy levels of the same family are involved in the avoided crossings when the electric field removes the electron from the dot occupied by the hole. For the dot couple the energy level $1 a$ is avoided crossed by energy level $1 b$ 


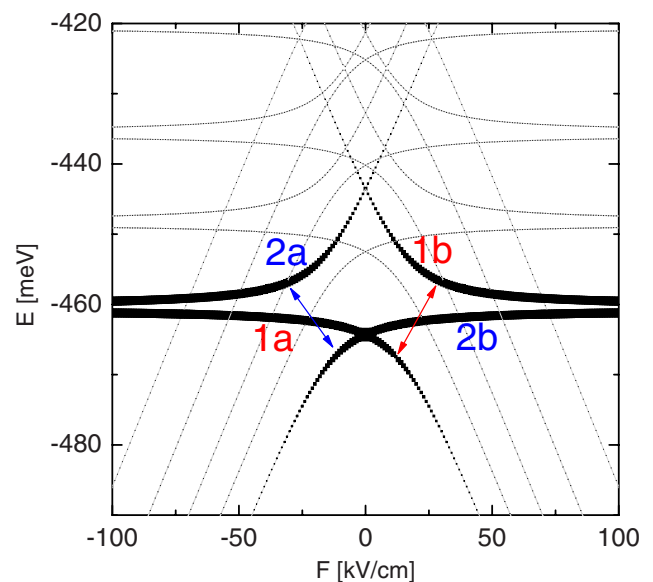

FIG. 2. (Color online) Exciton energy spectrum for two identical dots separated by barrier thickness of $5 \mathrm{~nm}$. The thickness of the lines is proportional to the recombination probability [see Eq. (7)]. The bright energy levels are denoted by the number 1 or 2 which indicates the dot in which the hole is localized (see Fig. 1). The two arrows indicate the energy levels that avoided cross when the electron is removed of the dot in which the hole is localized.

(see the avoided crossings marked by arrows in Fig. 2) when the electron is removed of the lower dot by a positive electric field $F>0$. A mirror reflection of this avoided crossings appears between the energy levels $2 a$ and $2 b$ at negative field. For intermediate coupling, i.e., for negligible hole tunneling, the energy levels of different families cross (see the crossing of $1 a$ and $2 b$ energy levels, as well as the crossing of $2 a$ and $1 b$ levels, both occurring at $F=0$ in Fig. 2). In the ground state at $F=0$ such a crossing leads to the ground-state degeneracy which is due to a negligible hole tunneling. The problem of the ground-state degeneracy for three and four identical dots is discussed below.

\section{A. Three identical dots: Intermediate coupling}

Figure 3(a) shows the energy spectrum for three identical dots separated by tunnel barriers of $4 \mathrm{~nm}$ in thickness. The spectrum is perfectly symmetric with respect to the electric field orientation and we will discuss only $F>0$. For $F=0$ the ground state $(2 b)$ is nondegenerate-in contrast to the twodot case of Fig. 2-and corresponds to the hole localized in the central dot [see the hole density plotted in Fig. 4(b)]. ${ }^{33}$ The electron occupies predominantly the same dot, but tunneling to the neighbor dots is visible [see Fig. 4(a)]. For a noninteracting electron-hole pair and negligible hole tunneling the ground state is threefold degenerate. Preference of the electron to stay inside the central dot and the electron-hole interaction leads to an effectively increased depth of the central dot for the hole which lifts the ground-state degeneracy.

For $F$ increased from 0 to $10 \mathrm{kV} / \mathrm{cm}$ the electron density corresponding to the $2 b$ level is removed from the lowest dot and increased in the uppermost dot [Fig. 4(c)]. The $2 b$ level becomes dark near $F=50 \mathrm{kV} / \mathrm{cm}$ [Fig. 3(a)] which is due to the separation of the carriers [see Figs. 4(e) and 4(g)]. The dissociation of the exciton in the $2 b$ energy level at $F>0$ occurs via an avoided crossing with the $2 c$ energy level. The
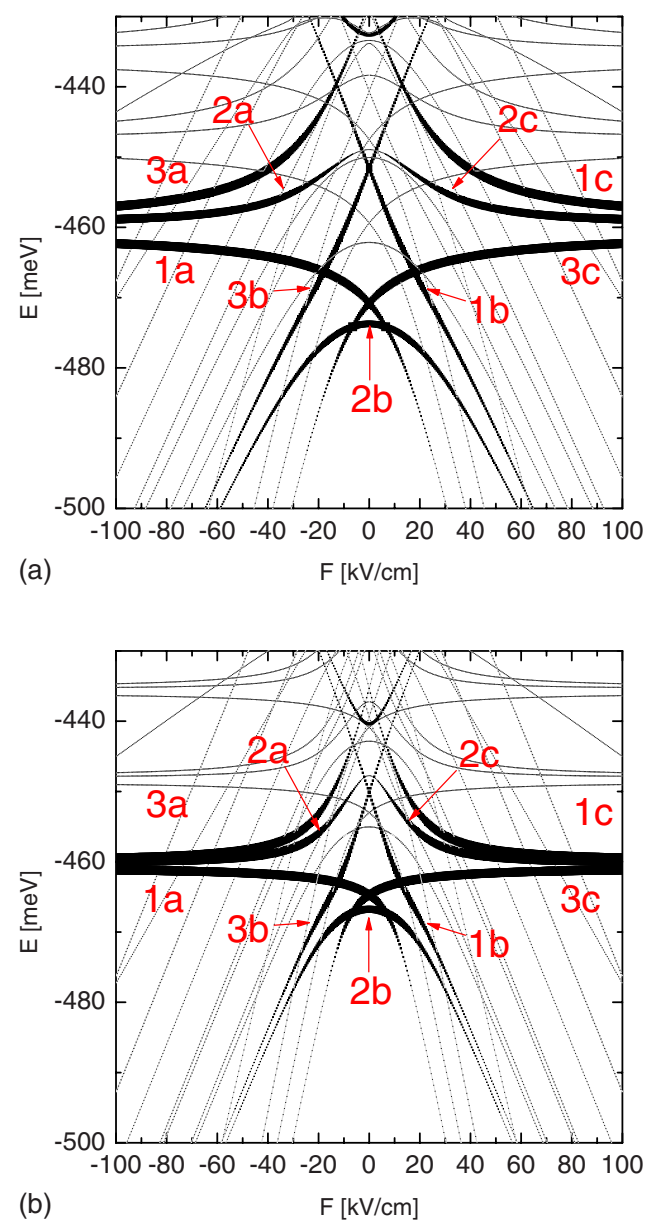

FIG. 3. (Color online) Exciton energy spectrum for three identical dots separated by barrier thicknesses of 4 (a) and $5 \mathrm{~nm}$ (b). The thickness of the lines is proportional to the recombination probability [Eq. (8)]. The bright energy levels are denoted by the numbers 1 , 2 , or 3 which indicates the dot in which the hole is localized (see Fig. 1). Letters $(a, b, c)$ mark the bright energy levels of the same family (with the hole localized in the same dot) that appear at $F$ $\ll 0, F \simeq 0$, and $F \gg 0$, respectively.

electron in the $2 c$ energy level [see Figs. 4(f) and 4(h)] enters the central dot occupied by the hole when electron density of $2 b$ gets localized by the field in the uppermost dot [see Figs. 4(f) and 4(h)].

Figure 5(a) shows that in the $3 c$ energy level for small field a residual presence of the electron in the lowest dot (1) is observed. As the field increases the electron becomes completely localized in the third dot (where the hole is localized). This results in an increased intensity of the $3 c$ line with respect to $F=0$ that can be noticed in Fig. 3(a). The $3 x$ family has a more interesting behavior for $F<0$, and for a symmetric dot this is a mirror reflection of the phenomena observed for the $1 x$ family at $F>0$. The energy levels $1 a$ and $3 c$ are degenerate at $F=0$. At low field in the $1 a$ state the electron density is a reflection of the $3 c$ density with respect to the central dot [see Fig. 6(a) and compare it to Fig. 5(a)]. In the $1 a$ energy level at $F=0$ the electron-hole distribution has a nonzero electric dipole moment in contrast to the $2 b$ energy level. As a consequence, the $1 a$ energy level reacts to 

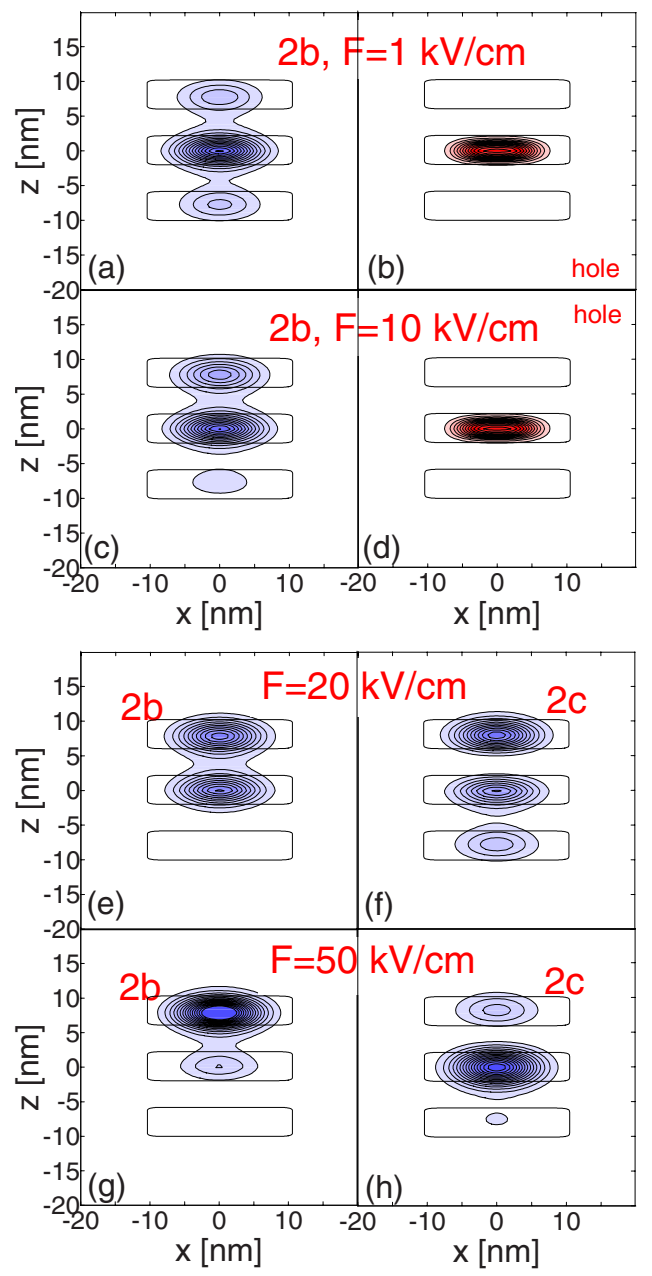

FIG. 4. (Color online) Cross section $(y=0)$ of electron [(a), (c), and (e)-(h)] and hole [(b) and (d)] densities in the states of the $2 x$ family, in which the hole is localized in the central (2) dot for three identical dots with interdot barrier thickness of $4 \mathrm{~nm}$ for various values of the electric field $F$. The corresponding energy levels are plotted in Fig. 3(a). Thin solid boxes indicate the region in which the confinement potential is at least $10 \%$ of its maximal value with respect to the center of each dot.

the field more strongly than $2 b$ [see Fig. 3(a)]. $1 a$ first becomes the ground-state energy level and then it becomes dark [see Fig. 3(a)] when the electron is removed from the lowest dot [see Figs. 6(b) and 6(c)]. At small electric field the electron in the $1 b$ energy level is localized mostly at the extreme dots of the triple stack [see Figs. 6(d)-6(f)]. At higher field the electron in the $1 b$ energy level becomes localized in the middle dot (the hole remains in the lowest dot in all the states of the $1 x$ family) and consequently the energy level becomes dark. Note that the slope of the $1 b$ energy level when it becomes dark is twice smaller than for the $1 a$ ground state when it is dark. This is due to the electric dipole moment which is twice smaller in $1 b$ (hole in the lowest dot and electron in the middle) than in the ground state $1 a$ (hole in the lowest dot and electron in the uppermost). At high field the electron becomes localized in the lowest dot only in the third state $(1 c)$ of the $1 x$ family [see Figs. 6(h) and 6(i)].

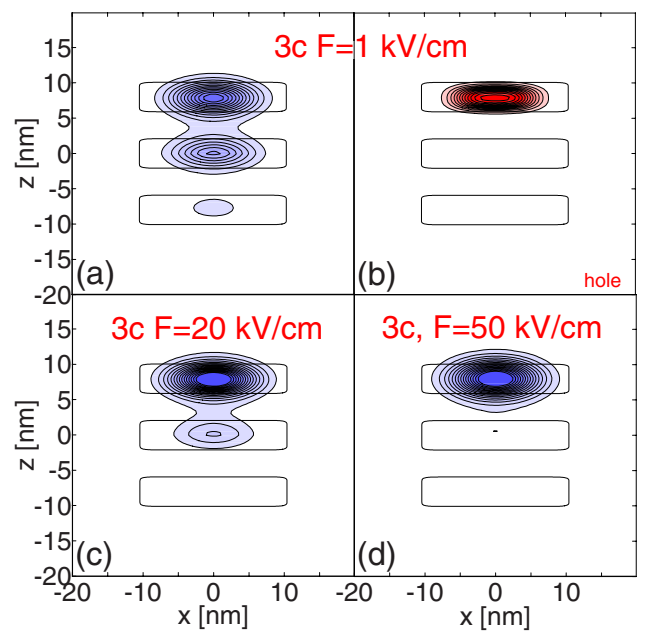

FIG. 5. (Color online) Same as Fig. 4 but for the $3 c$ energy level of Fig. 3. Plots [(a), (c), and (d)] show the electron density and (b) the hole density.

A strong electric field removes the electron tunnel coupling between the dots, so the electron becomes localized in a single dot similarly as the hole. In all the strong-field bright states $1 c, 2 c$, and $3 c$ the decrease of the electron energy with the electric field is compensated by the increase of the hole energy. As a consequence, at large $F$ all the bright levels $1 c$, $2 c$, and $3 c$ become degenerate. For increased barrier thickness to $5 \mathrm{~nm}$ degeneracy appears for smaller $F$ [see Fig. 3(b)].

The change of the spectrum with the addition of the third dot to the two-dot stack can now be summarized by comparison of Figs. 2 and 3. In the family $1 x$-associated with the hole localized in the lowest dot of the stack - the bright energy levels appearing at strong negative $F \ll 0$ and strong positive $F \gg 0$ fields- $1 a$ and $1 c$, respectively, exhibit an avoided crossing (Fig. 3) like the $1 a$ and the $1 b$ energy levels for the double dot case (Fig. 2). Similarly as in the two-dot case this avoided crossing-related to the transfer of the electron from the lower to the upper dot-appears for $F$ $>0$. The new feature of the $1 x$ family for three dots is the appearance of an energy level $(1 b)$ which enters the $1 a-1 c$ avoided crossing nearly linearly and appears as bright in the range of electric fields which induces the avoided crossing. Family $3 x$ associated with the dot at the opposite extreme end of the stack is a mirror reflection of $1 x$. In comparison to the two-dot case a qualitatively new group of bright levels is the $2 x$ family, associated with the dot situated inside the stack. The energy level which appears as bright near $F=0$ $(2 b)$ has a parabolic dependence on $F$ and anticross the two other bright energy levels of the family: $1 a$ at $F<0$ and $1 c$ at $F>0$.

\section{B. Three nonidentical dots: Intermediate coupling}

Let us first consider the case when a constant "gradient" in the depth of the confinement potential is present within the stack [see the inset to Fig. 7(c)]. Figure 7 presents the results for the case that the depth of the lowest dot (number 1) is 


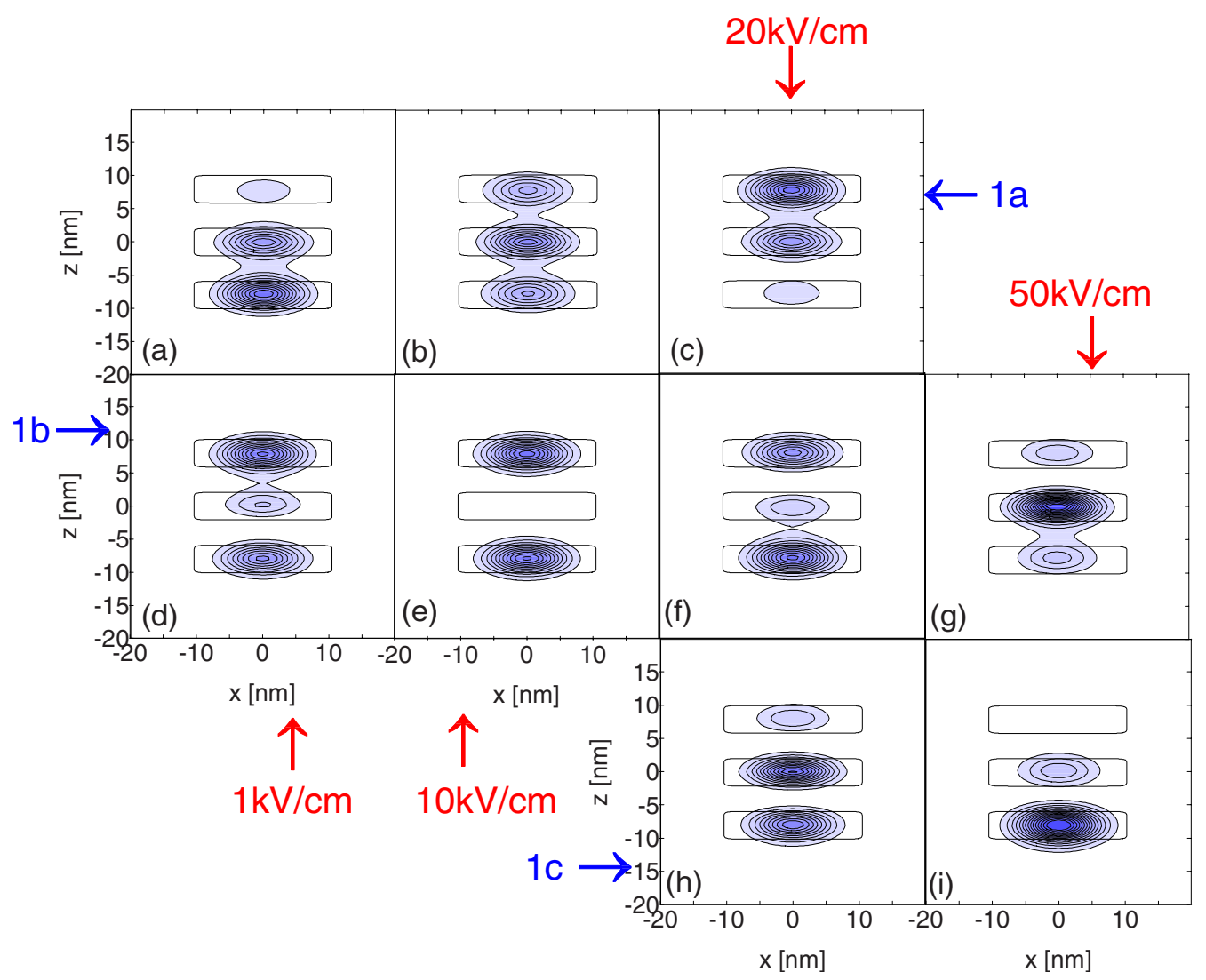

FIG. 6. (Color online) Same as Fig. 4 but for the $1 x$ family of states in which the hole is localized in the lowest dot. All the plots show the electron density. The upper [(a)-(c)], middle $[(\mathrm{d})-(\mathrm{g})]$, and lower $[(\mathrm{h})$ and (i) $]$ rows of plots correspond to $1 a, 1 b$, and $1 c$ energy levels of Fig. 3. The columns of plots from left to right correspond to $F=1,10,20$, and $50 \mathrm{kV} / \mathrm{cm}$.

increased by $10 \mathrm{meV}$ for both the electron and the hole while for the uppermost dot (number 3 ) it is decreased by the same amount for both carriers. The modification of the depth is directly translated into relative energy shifts between the families of energy levels. For large $F>0$, the $1 c, 2 c$, and $3 c$ energy levels are no longer degenerate but they are separated by roughly $20 \mathrm{meV}$ (sum of the confinement energy difference for both carriers in the separate dots).

The avoided crossings in the separate families are most clearly visible for weaker coupling with $t=6 \mathrm{~nm}$ in Fig. 7(c). Although the families of energy levels are shifted, the qualitative character of the avoided crossings within each family is the same as in the stack of identical dots. In the $2 x$ family a single avoided crossing is observed at both electric field orientations and appears when the electron is moved from the central to the upper or lower dot. On the other hand for the $1 x$ family only the positive electric field induces avoided crossings. The first avoided crossing corresponds to the removal of the electron from the lowest to the uppermost dot and the second from the lowest to the middle dot.

The spectrum in case the central dot is the deepest is plotted in Fig. 8 for $6 \mathrm{~nm}$ thick tunnel barriers (see the inset to Fig. 8, the depth of the central dot is increased by $10 \mathrm{meV}$, and the uppermost is decreased by $10 \mathrm{meV}$ ). Similarly as in the constant gradient case of Fig. 7(c) we see well resolved patterns of the three families of energy levels known from the results presented above. The families are shifted as expected from the varied potential depths.
In the discussed calculations for nonidentical dots we assumed that the depth of the dot is varied in the same way for both the electron and the hole. In Fig. 9 we show the spectrum calculated for equal electron confinement depths but with varied depths for the hole: $+20,0$, and $-20 \mathrm{meV}$ along the growth direction. The adopted shifts are equal to the sum of the electron and hole variation of Fig. 7(c). We see that both spectra presented in Figs. 7(c) and 9 are nearly identical. Same result is obtained for equal depth for the hole but varied potential depth for the electron. In our previous work $^{24}$ on the coupled two-dot system we demonstrated that the electron-hole interaction translates the asymmetry for one type of particle to an effective confinement potential for the other. This conclusion still holds for triple stacked dots.

\section{Three stacked dots: Strong coupling}

Figure 10(a) shows the spectra for a stack of triple identical dots separated by a $1 \mathrm{~nm}$ thin barrier. The strong coupling between the dots not only increases the electron energy splitting (which widens the spectrum in the energy scale) but also makes the electron states less susceptible to the manipulation by the external field. Therefore, in order to obtain an evolution of the spectrum comparable to the one observed in the intermediate-coupling case for $t=4 \mathrm{~nm}$ [Fig. 3(a)] both the electric field and the energy scale had to be increased by a factor of about 5. With this scaling the pattern of energy 

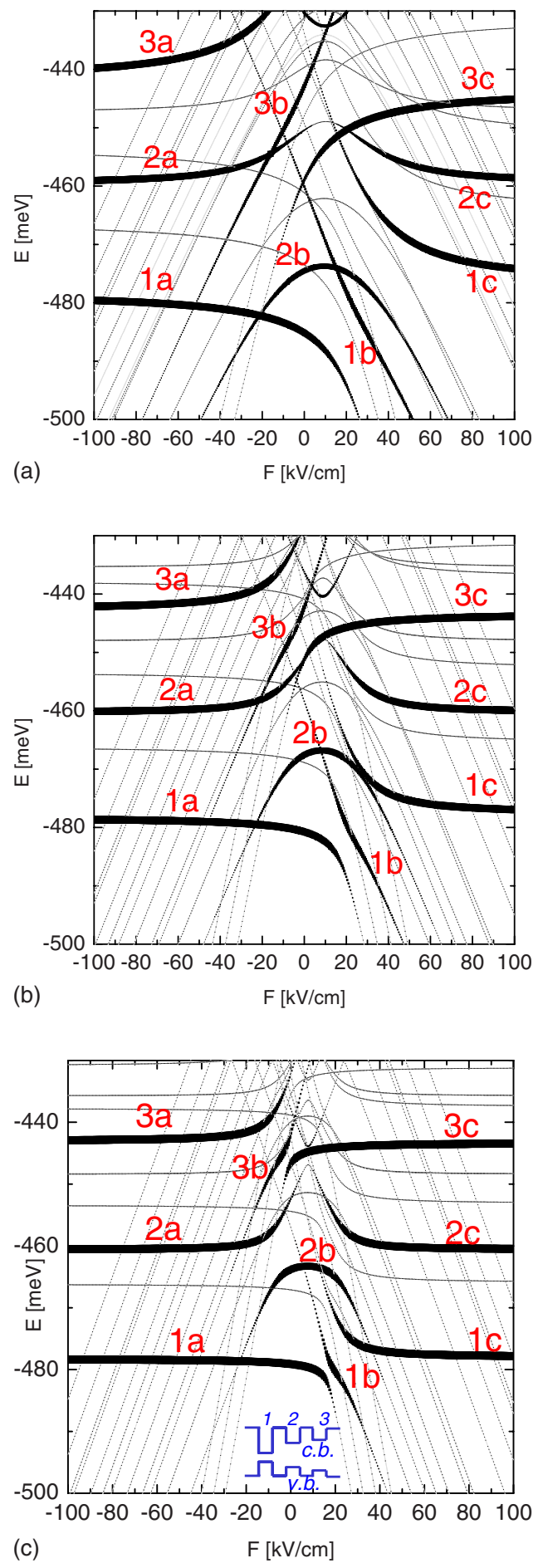

FIG. 7. (Color online) Same as Fig. 3 but for three nonidentical dots separated by barrier thicknesses of 4 (a), 5 (b), and $6 \mathrm{~nm}$ (c). The depth of the confinement potential of the dots 1,2 , and 3 is varied by $+10,0$, and $-10 \mathrm{meV}$ (constant gradient of potential depth), respectively, for both the electron and the hole. Schematic drawing of the valence and conduction band along the stack is presented in the inset of (c).

levels is similar to the intermediate-coupling spectrum [Fig. $3(\mathrm{a})$ ]. A specific feature of the strong-coupling case occurs for relatively small electric fields [see an enlarged picture

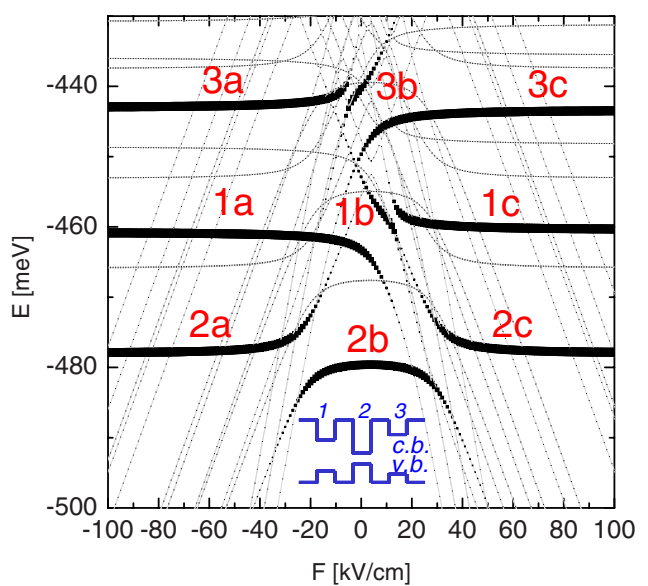

FIG. 8. (Color online) Same as Fig. 7(c) but for varied depths of the dot with barrier thickness of $t=6 \mathrm{~nm}$. The depth of the confinement potential of the dots 1,2 , and 3 is varied by $0,+10$, and $-10 \mathrm{meV}$, respectively, for both the electron and the hole. The inset shows a sketch of conduction and valence band extrema along the growth direction.

plotted in Fig. 10(b)] and consists in avoided crossings that occur between energy levels of different families. In Fig. 10(b) there are two such avoided crossings-marked by dashed rectangles. In the ground state the avoided crossing involves $1 a, 2 b$, and $3 c$ bright energy levels. Electron and hole densities for that avoided crossing are plotted in Figs. $11(\mathrm{~m})-11(\mathrm{r})$ for $F=0$ and in Figs. 11(s)-11(y) for $F$ $=10 \mathrm{kV} / \mathrm{cm}$. At $F=0$ all the states share nearly the same electron density [cf. Figs. 11(m), 11(o), and 11(q)] with a maximum in the central dot but with a strong penetration to the other two dots.

In the strong-coupling limit a simple product of the electron and hole single-particle wave functions [instead of a linear combination of such products—-see Eq. (4)] is a rela-

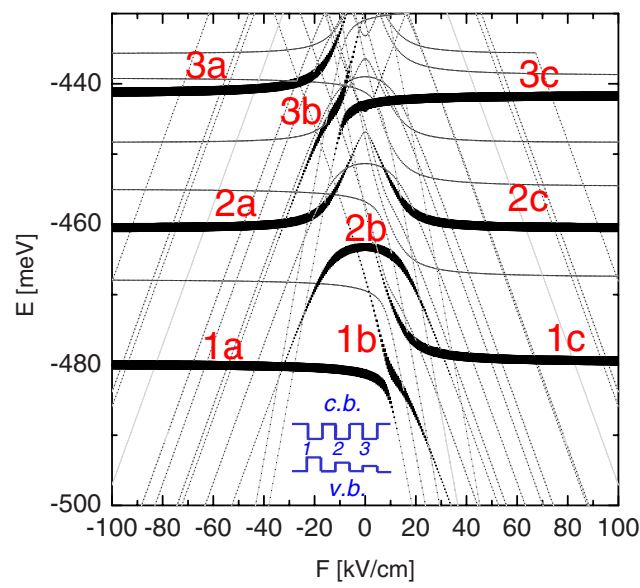

FIG. 9. (Color online) Same as Fig. 7(c) but for varied depths of the dot with barrier thickness of $t=6 \mathrm{~nm}$. The depth of the confinement potential of the dots 1,2 , and 3 is varied by $+20,0$, and $-20 \mathrm{meV}$, respectively, for the hole. Dots have equal depth for the electron. The inset shows a sketch of conduction and valence band extrema along the growth direction. 

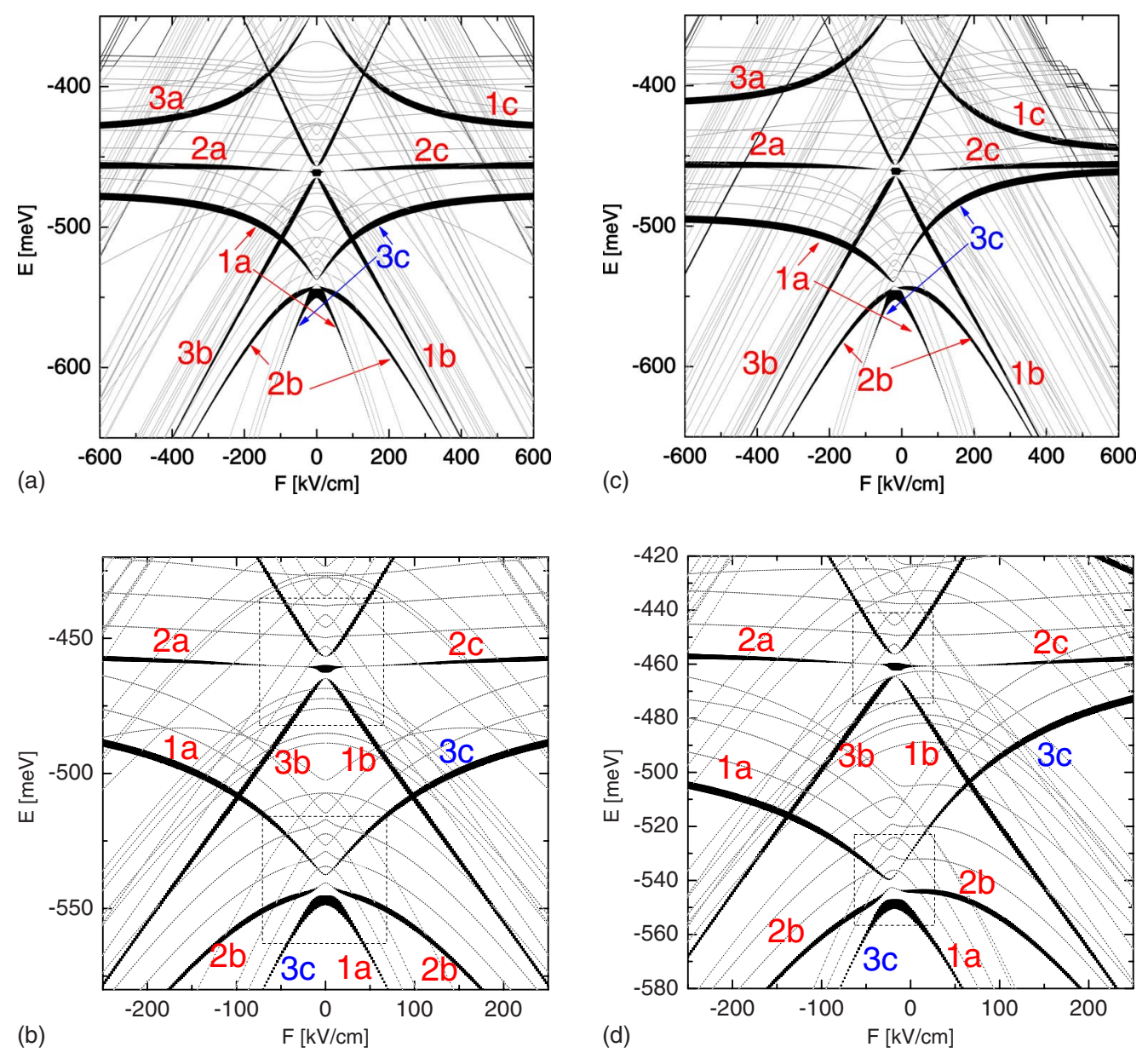

FIG. 10. (Color online) Spectra for the strong-coupling case with $t=1 \mathrm{~nm}$ (a) correspond to identical dots and in (c) the depth of the confinement potentials of the dots 1,2 , and 3 is modified by $+10 \mathrm{meV}$, and $-10 \mathrm{meV}$, respectively. (b) and (d) present fragments of (a) and (c), respectively, in which avoided crossings related to the avoided crossings between different families are observed.

tively good approximation for the exciton wave function. Therefore, although in principle only the total parity of the exciton state is a good quantum number at $F=0$, in the strong-coupling limit (and only in the strong-coupling limit) the states acquire also approximate single-particle parities (as discussed in Ref. 34). Below, we will refer to the singleparticle states and parities for the interpretation of the avoided crossings related to the hole tunneling in the strongcoupling limit. In all the three states involved in the $1 a$ $-2 b-3 c$ avoided crossing [the lower rectangle in Fig. 10(b)] the electron is in an even-parity state [see the electron density plot in Figs. 11(m), 11(o), and 11(q)], i.e., in its lowestenergy state. In the ground state the hole has a similar density [Fig. 11(r)] but with a weaker penetration to the top and bottom dots. The first excited state corresponds to a hole excitation, i.e., to an antibinding hole state [Fig. 11(p)]. At $F=0$ the hole in the first excited state occupies an odd-parity energy level. Due to the odd parity the hole density is bound to vanish in the center of the central dot. We see that, in fact, the hole is totally removed from the central dot [Fig. 11(p)]. The total envelope exciton wave function for this state is odd and therefore the recombination is strictly forbidden since the integral (7) vanishes. For a product of single-particle wave functions $\phi\left(\mathbf{r}_{\mathbf{e}}, \mathbf{r}_{\mathbf{h}}\right)=f_{e}\left(\mathbf{r}_{\mathbf{e}}\right) f_{h}\left(\mathbf{r}_{\mathbf{h}}\right)$ the formula for the recombination probability (7) reduces to

$$
p=\left|\int d^{3} \mathbf{r} f_{e}(\mathbf{r}) f_{h}(\mathbf{r})\right|^{2}
$$

i.e., to the square of the overlap between the hole and electron wave functions. For opposite parities of the two functions the overlap is strictly zero. For the second excited energy level the hole is an even state again [Fig. 11(n)] and the recombination probability is therefore nonzero but turns out to be small, about 15 times smaller as compared to the ground state. In the second excited state at $F=0$ the hole wave function changes sign in both barriers. The hole is more probable to be found at the extreme dots of the stack while the electron wave function is maximal in the central dot. This, along with the sign oscillations of the hole wave function, makes the electron and hole wave functions nearly orthogonal, hence the small recombination probability. Note that the electron and hole densities presented in Figs. 11(m) and $11(\mathrm{r})$ are in good qualitative agreement with the calculations for $F=0$ presented in Fig. 5 of Ref. 4 for the electron 

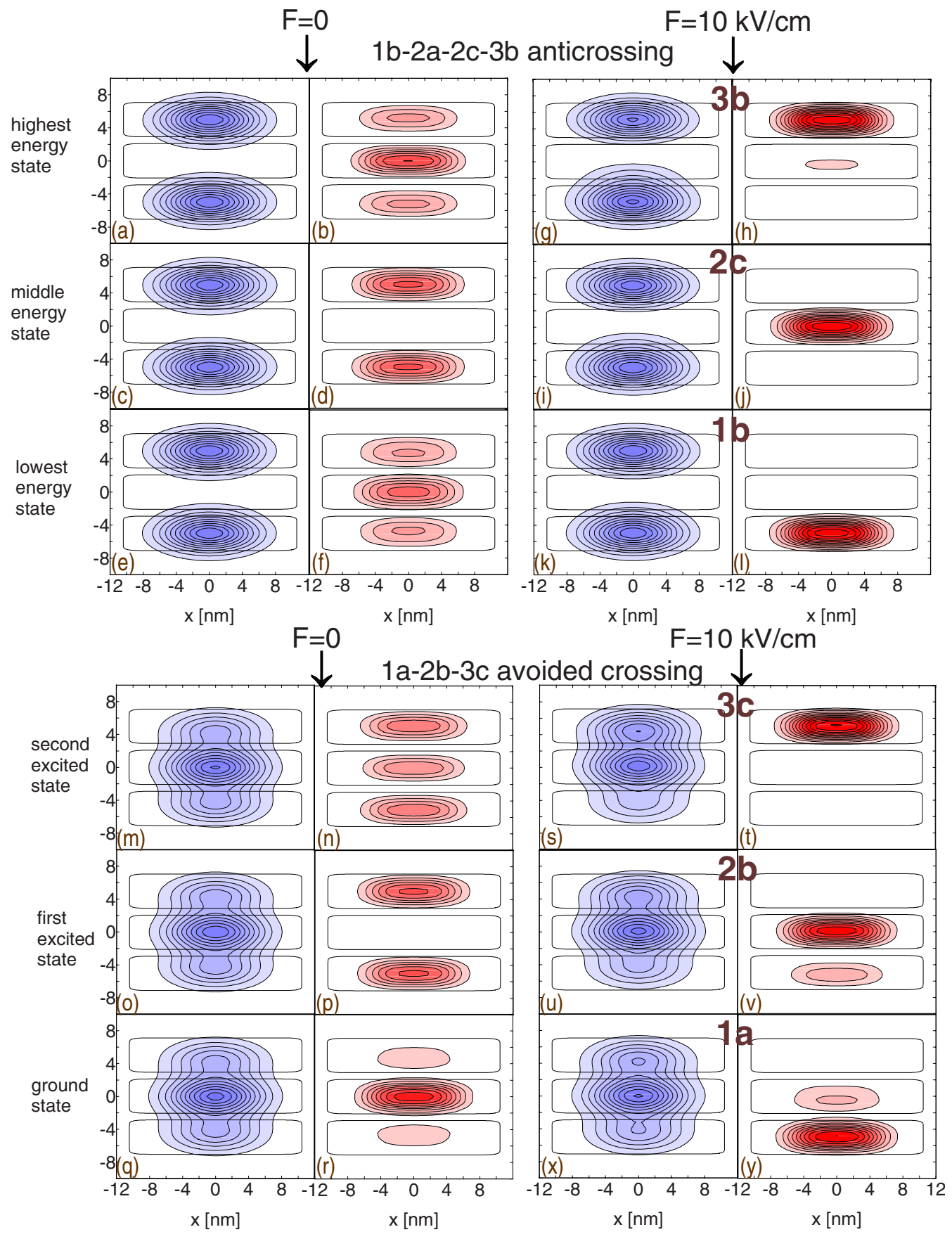

FIG. 11. (Color online) Cross section $(y=0)$ of the electron and hole densities for the energy levels participating in the two avoided crossings presented in Fig. 10(b). The upper group of plots [(a)-(1)] corresponds to the avoided crossing marked by the upper rectangle in Fig. 10(b) and the lower group $[(\mathrm{m})-(\mathrm{y})]$ to the ground-state avoided crossing marked by the lower rectangle in Fig. 10(b). In each group-higher-energy states correspond to the upper panels. The first (second) column of plots shows the electron (hole) density at $F=0$ (namely, a,c,e,m,o,q for electron, and b,d,f,n,p,r for the hole). The third (fourth) the electron (hole) density at $F=10 \mathrm{kV} / \mathrm{cm}$ (g,i,k,s,u,x for electron, and h,j,l,t,v,y, for the hole). Identities of the states when their energy levels emerge from the avoided crossing can be deduced from the plots for $F=10 \mathrm{kV} / \mathrm{cm}$. The densities are therefore labeled according to the "family" notation used in previous discussion.

and hole states in a stack of three strongly coupled dots each of different size and shape.

When the electric field is switched on the recombination probability of the ground state is reduced and all the three energy levels leave the avoided crossing region [lower rectangle in Fig. 10(b)] as bright states with comparable recombination probabilities. The electron and hole densities for these energy levels are plotted for $F=10 \mathrm{kV} / \mathrm{cm}$ in Figs. 11(s) $-11(\mathrm{y})$. Already for this field the hole tunnel coupling is nearly extinguished [in Figs. 11(t), 11(v), and 11(y)]. When the hole becomes localized in the single dot, the discussion of energy levels in previously used terms of families becomes valid again. Therefore, outside the avoided crossing region we may identify the ground state as the $1 a$ energy 
level, the first excited bright state as $2 b$, and the second as $3 c$. Outside the discussed avoided crossing range of small electric fields, the energy levels of different families cross like in the intermediate-coupling case [see, for instance, the crossing of the $3 c$ and $1 b$ levels in Fig. 10(b)].

Let us now turn our attention to the second avoided crossing observed in Fig. 10(b) (the region marked with the upper dashed rectangle). From the negative $F$ side the avoided crossing occurs between the $1 b, 2 a$, and $3 b$ energy levels. At $F=0$ in all the states that enter this anticrossing the electron is in the first excited state of odd parity with respect to the center of the stack [see Figs. 11(a), 11(c), and 11(e)]. For symmetry reasons, the electron density in this state vanishes in the center of the stack, but not entirely in the central dot, since a leakage from the extreme dots is observed. At $F=0$, in the lowest of the three energy levels participating in the avoided crossing, the recombination is forbidden due to the even parity of the hole [opposite to the electron parity-see Fig. 11(f)]. For the same reason, at $F=0$ the recombination from the highest energy of the three anticrossing levels is strictly forbidden [for the hole density, see Fig. 11(b)]. Consequently only the middle-energy state is bright at $F=0$. Note the similarity of the electron and hole densities in the bright state at $F=0$ [compare Figs. 11(c) and 11(d)] both the particles are in the lowest-energy antibinding $l=0$ energy levels. For $F=10 \mathrm{kV} / \mathrm{cm}$ the hole coupling is removed [see Figs. 11(h), 11(j), and 11(1)]. The order of the bright energy levels when they leave the avoided crossing region is the same as in the ground-state avoided crossing, i.e., the lowestenergy bright level at $F>0$ is associated with the hole localized in dot 1 , the middle energy with dot 2 , and the energy level in which the hole is in dot 3 goes up with growing $F$.

In the intermediate-coupling region, when we varied the dot confinement potential, the families of energy levels shifted with respect to each other, as discussed in Sec. III B. The exciton spectrum for the case in which the potential depths are changed according to the constant gradient case (as discussed in Sec. III B) is plotted in Fig. 10(c). Comparison of Figs. 10(a) and 10(c) leads to the conclusion that the effect of the confinement variation on the spectrum for the strong coupling is distinctly less pronounced than for the intermediate-coupling case. This is due to the electron coupling effects that are much stronger as compared to the potential variation (see the beginning of Sec. III). In this sense the differences in dot confinement are masked by the strong electron tunnel coupling. The avoided crossings that appear for small fields due to the hole tunnel coupling are presented in detail in Fig. 10(d). For nonidentical dots they are no longer symmetric and they are shifted with respect to $F=0$ field, but they distinctly preserve their character [compare with the identical dots case of Fig. 10(b)]. In particular, in both avoided crossings marked by rectangles in Fig. 10(d) at the center of the avoided crossing a single energy level with a much stronger recombination probability than the others appears. Also the energy position of the brighter energy level within the triple of interacting energy levels is conserved. In the lowest-energy avoided crossing it is the lowest-energy level. In the second avoided crossing the brightest is the second energy level of the three interacting levels. Recombination of the two other levels is no longer forbidden by the

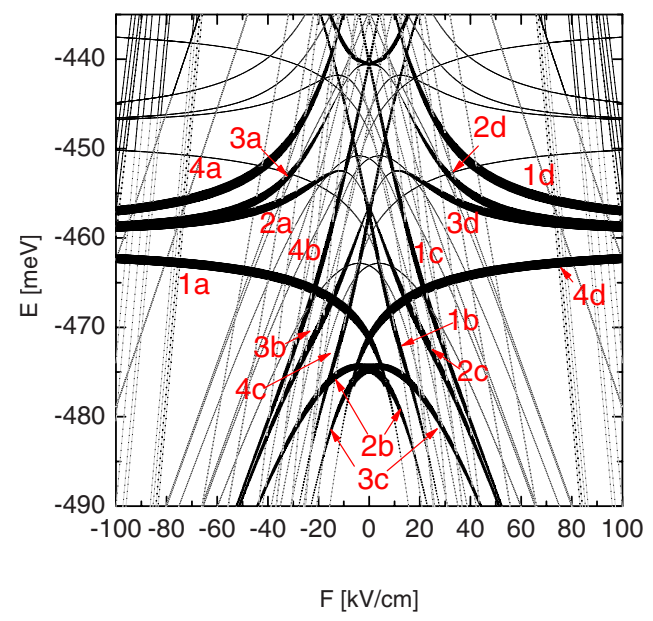

FIG. 12. (Color online) Same as Fig. 3(a) but for four identical dots separated by identical barriers of thickness $4 \mathrm{~nm}$.

symmetry-related selection rules but it remains small. The same rule applies for the next avoided crossing on an energy scale that did not fit into Fig. 10.

\section{Four identical dots: Intermediate coupling}

The spectrum for four identical dots separated by barriers of width $4 \mathrm{~nm}$ is presented in Fig. 12. At $F=0$ the ground state is twofold degenerate (like in the case of two-dot stacks-see Fig. 2-and in contrast to the stack of three dots-see Fig. 3). At $F=0$ in the ground state a crossing of $2 b$ and $3 c$ energy levels is obtained as a function of electric field. This degeneracy is due to the absence of hole tunneling and from the fact that for the electron both internal dots are equivalent. The electron densities in $2 b$ and $3 c$ energy levels at a weak electric field of $F=0.5 \mathrm{kV} / \mathrm{cm}$ can be inspected in Figs. 13(a) and 13(b), in which the dot occupied by the hole is marked by a red dot. In both the $2 b$ and $3 c$ states the electron is most probable to be found in the same dot as the hole. Electron tunneling to adjacent dots is also observed but with a clear preference for the neighbor dot that is situated inside the stack and not at its end. At a positive electric field the $2 b$ level goes down in energy (cf. Fig. 12) since the dipole moment of this state is aligned with the electric field [see Fig. 13(a) - the centers of the electron and hole distributions are displaced with respect to each other in the direction of the electric force]. Opposite is the effect of the field on the $3 c$ level in which the dipole moment is inverted. Due to the carrier distribution presented in Figs. 13(a) and 13(b) both these states have nonzero intrinsic dipole moments, hence the shifts of the maxima of their energy levels off $F$ $=0$ : to negative $F$ for $2 b$, and to positive $F$ for $3 c$.

At $F=0$ the first excited state is twofold degenerate ( $1 a$ and $4 d$ energy levels - see Fig. 12) and corresponds to the hole entirely localized in the extreme dots of the stack. The electron density in these states is maximal in the extreme dots but extends to the interior of the stack [see Figs. 13(c) and $13(\mathrm{~d})]$.

The electron densities plotted in Figs. 13(f)-13(j) for $F$ $=40 \mathrm{kV} / \mathrm{cm}$ correspond to the energy levels $3 d, 2 d$, and $1 d$ 


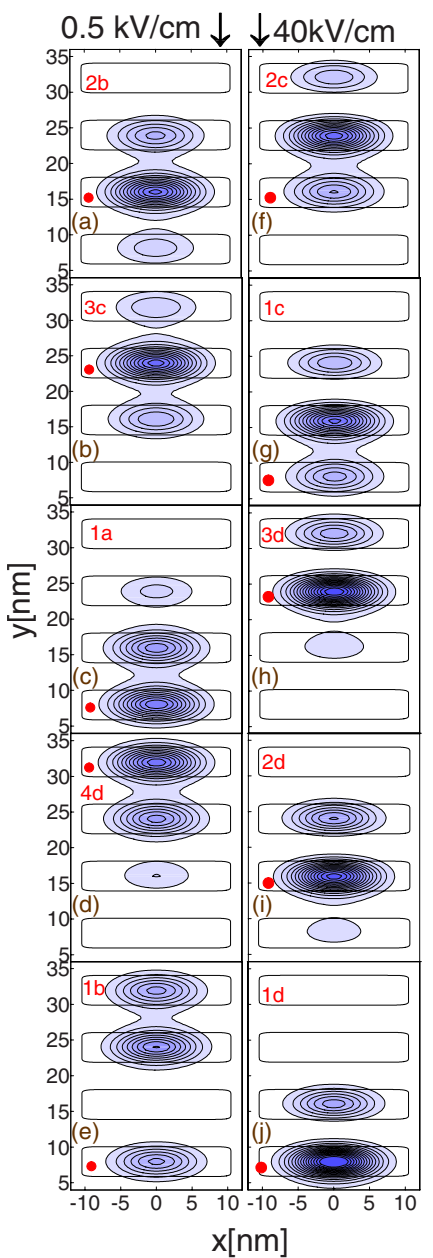

FIG. 13. (Color online) Electron densities for the stack of four identical dots (for the energy levels presented in Fig. 12). The left column of plots [(a)-(e)] corresponds to $F=0.5 \mathrm{kV} / \mathrm{cm}$ and the right column $[(\mathrm{f})-(\mathrm{j})]$ to $F=40 \mathrm{kV} / \mathrm{cm}$. In each case the hole is totally localized within the dot specified by the family label (additionally marked a dot).

which remain bright at $F \gg 0$ as well as $1 c 2 c$ levels which appear as temporarily bright within the avoided crossings of $1 b-1 d$ the $2 b-2 d$ energy levels, respectively.

Comparing the four-dot spectrum of Fig. 12 with its counterpart for three dots Fig. 3 we notice that in the $1 x$ familyassociated with the dot at the end of the stack-addition of a fourth dot results in the appearance of a second level $1 c$ in addition to $1 b$ that depends nearly linearly on the field becomes bright when entering the avoided crossing between the energy levels that remain bright at strong negative and positive fields in the four-dot case these are labeled by $1 a 1 d$, respectively.

For three dots a member of the $2 x$ family $(2 b)$ associated with the internal dot of the stack was the nondegenerate ground state at $F=0$. It entered a single avoided crossing with $2 a$ (the bright level at $F \ll 0$ ) $2 c$ (the bright one at $F$ $\gg 0$ ). Consequently $2 b$ level was a symmetric function of $F$. For four dots in the $2 x$ family an energy level appears that is temporarily bright at $F>0$ with a nearly linear dependence on $F$. For four dots this level is labeled by $2 c$. Due to its interaction with the $2 c$ level the "parabolic" $2 b$ energy level looses its symmetry with respect to $F=0$ becomes a deformed parabola as a function of field.

\section{E. Four nonidentical dots: Intermediate coupling}

Figure 14(a) shows the spectrum for a stack of four dots with a gradient of the depth of the confinement potentialthe depths of the dots from the lowest to the uppermost are increased for both carriers by $20,10,0,-10 \mathrm{meV}$, respectively [see the schematic drawing of the confinement potential above Fig. 14(a)]. Near $F=0$ the ground state is the bright energy level with the carriers confined in the deepest dot number 1 . The ground-state dissociation appears with $1 a-1 b$ avoided crossings near $F=15 \mathrm{kV} / \mathrm{cm}$. For higher electric field the ground state is dark and corresponds to the electron in dot 4. The lowest bright energy level between 15 and $20 \mathrm{kV} / \mathrm{cm}$ is $1 b$. For $F>20 \mathrm{kV} / \mathrm{cm}$ the electron in $1 b$ becomes localized in dot 3 and the energy level becomes dark. We notice the reduced slope of the energy level $1 b$ when it becomes dark compared to the dark ground state. The subsequent bright energy level $1 c$ becomes dark with the electron localized in dot 2 which is adjacent to the dot occupied by the hole. Consequently the dipole moment slope of the energy level as function of $F$ is still smaller. For the same reason we notice that in $1 c$ the electron-hole pair is significantly more resistant to dissociation as compared to $1 b$ note the increased $F$ interval in which $1 c$ is bright.

In the $1 x$ family all the avoided crossings of the bright energy levels appear at positive $F$ which tends to remove the electron from the lowest dot. On the other hand in the $2 x$ family the electron is removed by negative $F$ to dot 1 producing a wide $2 a-2 b$ avoided crossing similarly as in the stack of three dots near $F=-10 \mathrm{kV} / \mathrm{cm}$. For positive field two avoided crossings are observed. The first one $2 b-2 c$ appears when the electron is removed to dot 4 , and the second one $2 c-2 d$ when the electron is removed to dot 3 . Note that the slope of the dark energy level that comes from the $2 b-2 c$ avoided crossing is parallel to the dark part of the $1 b$ energy level. In both cases the hole electron are separated by an empty dot. Similarly the $2 c$ energy level when dark is parallel to the dark $1 c$ energy level since the carriers occupy adjacent dots.

The avoided crossing pattern in the $4 x$ family is clearly an inverted shifted pattern of the $1 x$ family. The inversion is due to the hole localized at the other extremity of the stack the shift to the reduced depth of dot 4 . Similarly the $3 x$ family is a shifted inverted counterpart of the $2 x$ family.

Figure 14(b) shows the spectrum for varied depths of the dots. Dot 2 is deepest with depth increased by $20 \mathrm{meV}$ for both carriers from the values given in Sec. II see the schematic potential profile above the figure. The analogous shifts for dots 1,3 , and 4 are $10,-10$, and $0 \mathrm{meV}$, respectively. The patterns of the avoided crossings remain characteristic to the position of the dot in which the hole is localized within the stack [cf. Fig. 14(a)]. More pronounced deformation appears only in the $3 x$ family associated with the dot which is the shallowest is visible in a modified shape of the $3 b$ bright line. 

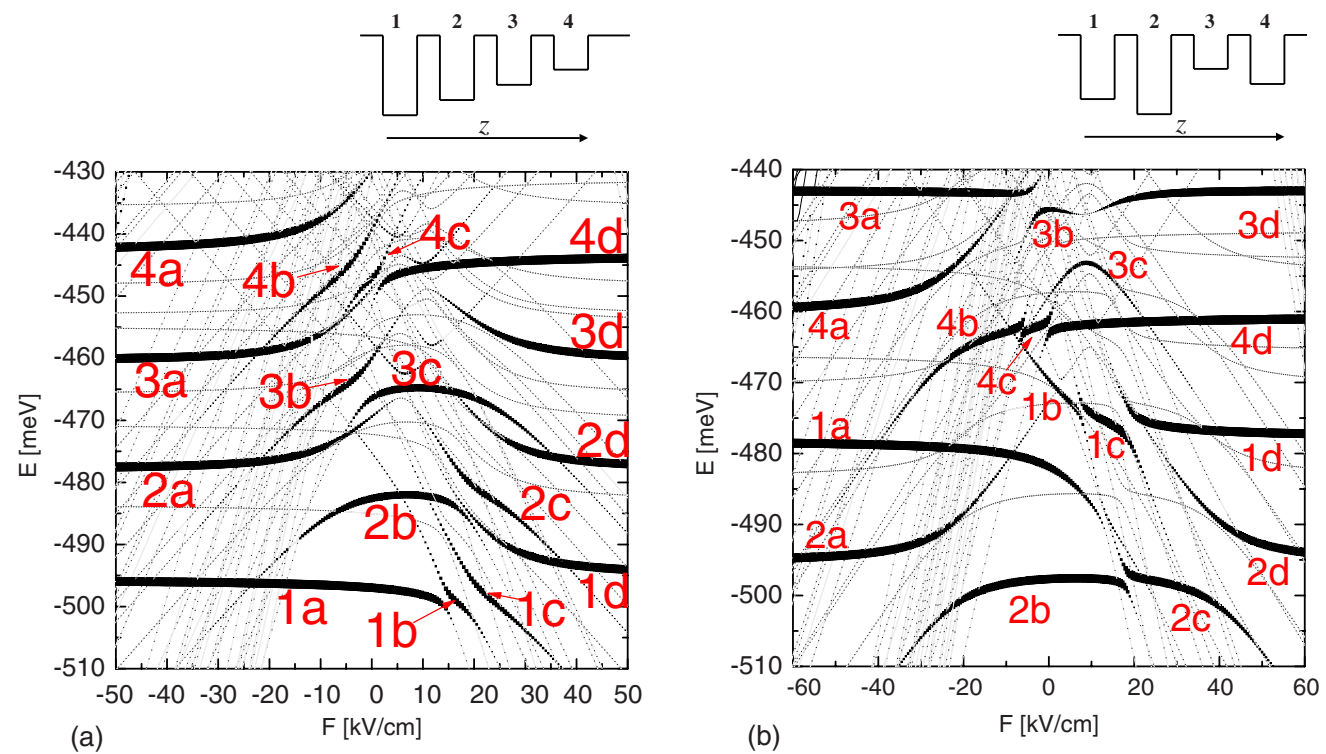

FIG. 14. (Color online) The spectrum for four stacked dots for barrier thickness of $5.5 \mathrm{~nm}$. In (a) a constant gradient of the confinement potential depths of the dots is assumed: (see the schematic drawing above the plot) the depth of each subsequent dot is decreased by 10 meV for both the electron and the hole when one moves up the stack. In (b) it is assumed that the shifts of the dot depth for both carriers are from the bottom to the top of the stack: $+10,+20,-10$, and $0 \mathrm{meV}$.

\section{F. Four dots: Strong coupling}

Similarly to the three dot case the spectrum for four dots in the strong-coupling regime [see Fig. 15(a)] resembles the one of the intermediate-coupling case see Fig. 12. Like for three strongly coupled dots (Sec. III C) qualitative differences appear for small $F$ where avoided crossings of states of different families are obtained. The region of the two-lowest avoided crossing is shown in more detail in Fig. 15(b) with the hole electron densities plotted in Fig. 16. In the ground state we have avoided crossing of $1 a 2 a, 3 c$, and $4 d$ energy levels. In the center of the avoided crossing near $F=0$ the ground state becomes distinctly brighter than the three other levels. The hole electron density in these states look alike [see Fig. 16(h)] with the exception of weaker hole tunneling. As a matter of fact at $F=0$ the electron densities are nearly identical for all the four energy levels [see Figs. 16(e)-16(h)]. In all these energy levels the electron is in an even-parity single-particle state. In the first excited state the hole state is odd so recombination is forbidden [see Fig. $16(\mathrm{~g})$ and Eq. (8)]. For the same reason it is forbidden for the third excited state [see Fig. 16(e)]. The recombination is allowed for the second excited state [see Fig. 16(f)] fin which the hole is in an even-parity state. The recombination probability is nonzero but small since the hole occupies only dots at the top the bottom of the stack while the electron occupies mostly the internal ones.

An electric field of $F=20 \mathrm{kV} / \mathrm{cm}$ is nearly strong enough to break the hole tunnel coupling which results in the hole localization in a single dot.

In the second avoided crossing that appears higher in energy involves the $1 b, 2 c, 3 b$, and $4 c$ energy levels the electron at $F=0$ occupies an odd-parity state with probability maxima at the ends of the stack [see Figs. 16(a)-16(d)]. The parity of the hole in the subsequent energy levels changes like in the lower avoided crossing: it is even in the lowestenergy state, odd in the second-energy state, etc. Recombination is not forbidden by symmetry from the second- [Fig. 16(c)] and fourth- [Fig. 16(a)] -energy states. It is the second energy level which is the brightest, since then the hole density is maximal in the outer dots like the electron density. In this state both the electron and the hole are in their first single-particle excited states. As a general rule the largest recombination probability is obtained for the cases that the electron and the hole excitation are the same. As an additional illustration, in Fig. 15(a) we notice that in the third avoided crossing (associated with the third single-electron state) it is the third-energy state that is the brightest at $F=0$.

The low-field spectrum for a system of nonidentical dots is presented in Fig. 15(c). The lowest dot depth is increased by $20 \mathrm{meV}$, the second by $10 \mathrm{meV}$, and the uppermost is decreased by $10 \mathrm{meV}$ (constant gradient). The avoided crossings related to mixing of the families are shifted from $F=0$ and deformed, i.e., not symmetric with respect to the center of the avoided crossing. For instance, in the lowest-energy bright state the left arm tends to the $4 d$ energy level when the hole tunneling is lifted and the right arm tends to $1 a$. For identical dots $4 d$ and $1 a$ are equivalent counterparts-the energy of $4 d$ at $F$ is equal to the energy of $1 a$ at $-F$. This is no longer the case for nonidentical dots, hence the asymmetry in the avoided crossing. The asymmetry is also observed in the recombination probability. In particular, the recombination probability of energy level $1 a$ vanishes with field in a slower way than in the $4 d$ energy level, which is a direct consequence of the difference in the depths of dots 1 and 4. In spite of the asymmetry in the avoided crossings the general features are similar to those observed for identical dots. A single energy level much brighter than the others appears in the center of the avoided crossings. 

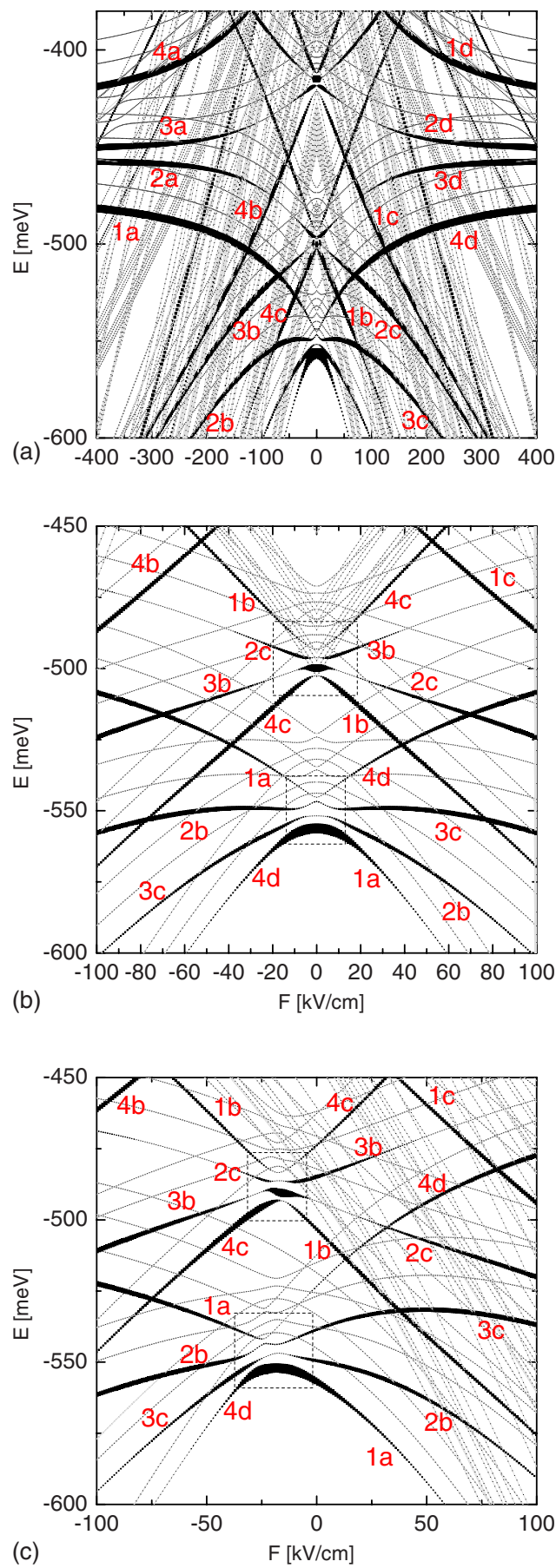

FIG. 15. (Color online) Spectra for four dots with barrier width of $1 \mathrm{~nm}$; (a) and (b) correspond to identical dots and in (c) the depth of the confinement potentials of the dots $1-4$ is varied by $+10,+0$, -10 , and $-20 \mathrm{meV}$, respectively. (b) is a zoom of (a).

\section{G. Simple modeling of avoided crossings between different families}

The insight gained on the study of the multiple avoided crossings between energy levels of different families allows us to introduce a simple modeling, which may be useful, for instance, in the interpretation of experimental data. During the hole transfer the electron density changes only slightly

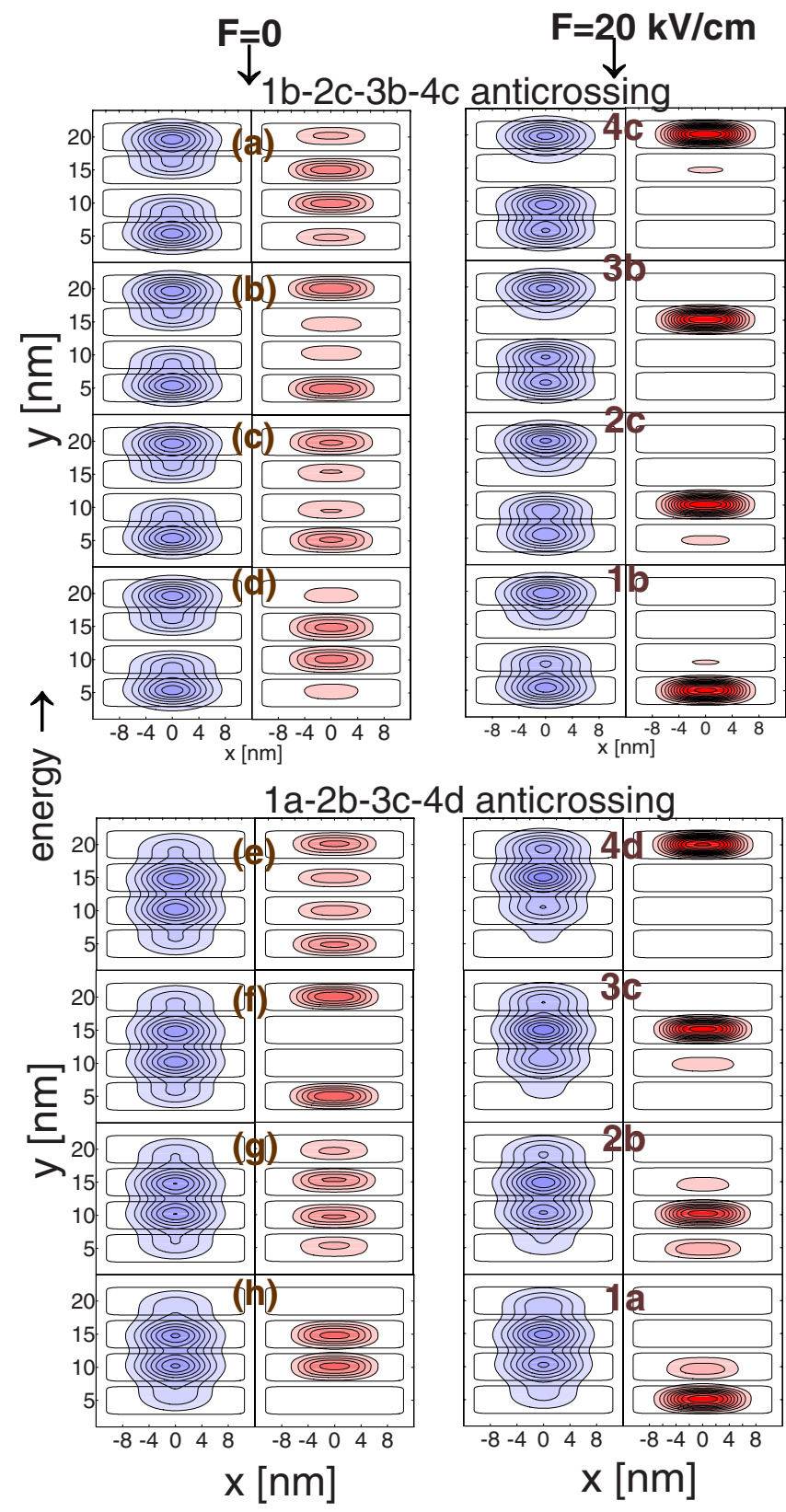

FIG. 16. (Color online) Cross section $(y=0)$ of the electron and hole densities corresponding to the energy levels participating in the two avoided crossings marked by rectangles in Fig. 15(b). The lower group of plots [the ones marked by (e)-(h) for $F=0$, and by the family labels $1 a, 2 b, 3 c$, and $4 d$ for $F=20 \mathrm{kV} / \mathrm{cm}$ ] corresponds to the ground-state avoided crossing marked by the lower rectangle in Fig. 15(b). The upper group of plots [(a)-(d) and $1 b, 2 c, 3 b$, and $4 c]$ ) corresponds to the avoided crossing marked by the upper rectangle in Fig. 15(b). In each group-the higher-energy states correspond to the upper panels. The first (second) column shows the electron (hole) density at $F=0$ and the third (fourth) the electron (hole) density at $F=20 \mathrm{kV} / \mathrm{cm}$. Identities of the states when their energy levels emerge from the avoided crossing can be deduced from the plots for $F=20 \mathrm{kV} / \mathrm{cm}$. The corresponding densities are therefore labeled in the family notation. 
(see Fig. 16), so we may focus our attention on the singlehole energy levels. We assume that with each dot we can associate a single localized wave function $\left(\psi_{i}\right)$. The multiple avoided crossing between different families can be modeled using superposition

$$
\psi_{\mu}=\sum_{i=1}^{4} d_{i}^{\mu} \psi_{i}
$$

For no tunnel coupling the wave function is orthogonal due to the lack of overlap. In presence of the coupling one can construct orthogonal wave functions each associated with a single dot and weakly penetrating the nearest neighbor dots with an inverted sign which will preserve their mutual orthogonality. For orthogonal wave functions the values of $d_{i}^{\mu}$ are found by diagonalization of the single-hole Hamiltonian $H_{h}$ in basis (9). A matrix element of the single-hole Hamiltonian is $H_{i j}=\left\langle\psi_{i}\left|H_{h}\right| \psi_{j}\right\rangle$, with

$$
H=\left(\begin{array}{cccc}
\epsilon-\frac{3}{2} F e d & c & 0 & 0 \\
c & \epsilon-\delta-\frac{1}{2} F e d & c & 0 \\
0 & c & \epsilon-\delta+\frac{1}{2} F e d & c \\
0 & 0 & c & \epsilon+\frac{3}{2} F e d
\end{array}\right) .
$$

The diagonal elements are equal to the sum of the single-dot confinement energy $\epsilon$ (in the following taken as the reference energy level $\epsilon=0$ ), the energy shift due to the electric field, and an additional energy shift $\delta$ introduced to account for the effect of the interaction with the electron which, in the lowest-energy avoided crossing is localized rather in the central two dots 2 and 3 ( $\delta>0$ - the central dots will be effectively deeper), and in the second avoided crossings stays rather in dots 1 and $4(\delta<0$-the central dots will be effectively shallower). In Eq. (10) the hole tunneling between the nearest neighbor dots is described by the hopping parameter $c=\left\langle\psi_{i}\left|H_{h}\right| \psi_{i+1}\right\rangle$. We assume $d=5 \mathrm{~nm}$ (interdot barrier thickness of $1 \mathrm{~nm}$ ) in accordance with Sec. IV. The eigenvalues of Hamiltonian (10) are plotted as functions of the electric field in Figs. 17(a)-17(c), for and $\delta=0, \delta=3 \mathrm{meV}$, and $\delta=-3 \mathrm{meV}$, respectively. In each plot we consider the case of negligible tunneling $(c=0)$ and $c=2.5 \mathrm{meV}$. For a pair of dots the splitting between the energy levels is given by $2 c$ $=5 \mathrm{meV}$, i.e., the value corresponding to the hole levels splitting at the barrier width of $1 \mathrm{~nm}$. For $c=0$ the energy levels are simply equal to the diagonal (single-dot) elements of the matrix (10) and cross. Therefore, we see that the avoided crossing is due to the tunneling of the hole between the nearest dots. Below we only discuss the results obtained for $c=2.5 \mathrm{meV}$. For $\delta=0$ the distances between the energy levels in the center of the avoided crossing $(F=0)$ are equal. This is not the case in any of the two avoided crossing presented in Fig. 15(b). In the lowest avoided crossing of Fig. 15(b) the third and fourth energy levels distinctly approach
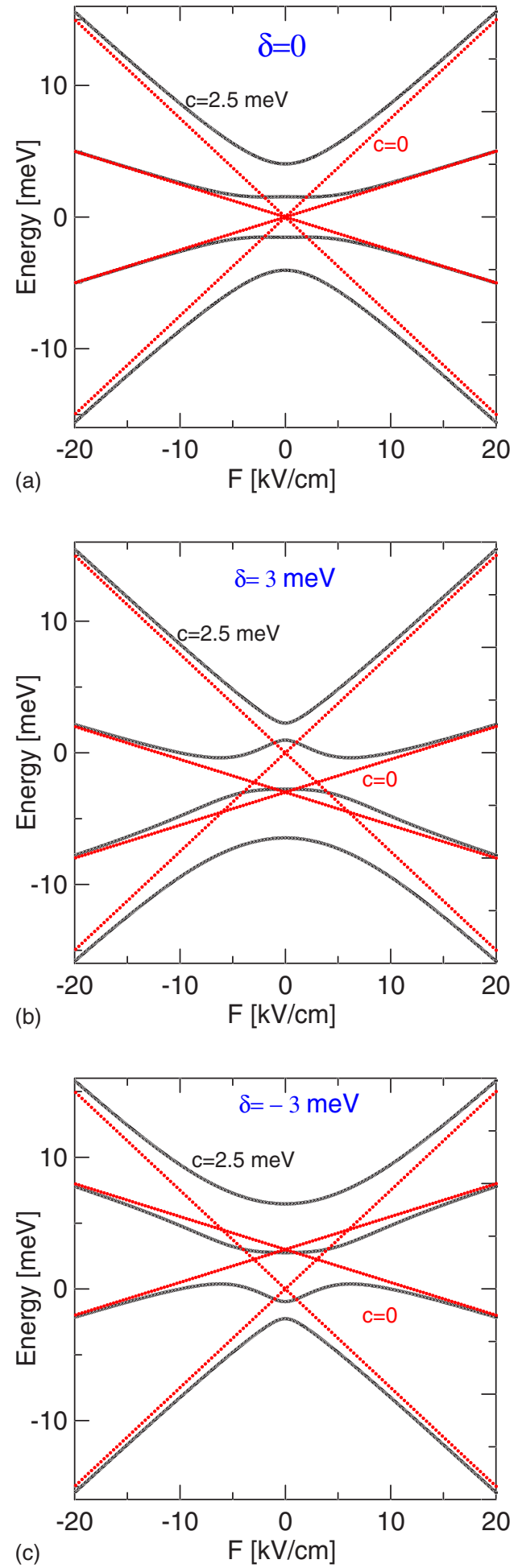

FIG. 17. (Color online) Energy eigenvalues of the hole Hamiltonian (10) modeling the multiple avoided crossing between energy levels of different families. Black lines correspond to the hopping matrix element of $c=2.5 \mathrm{meV}$, and red lines to the neglected hole tunneling $c=0$. In (a) all the dots are equivalent $(\delta=0)$, in (b) the two central dots are effectively deeper due to the interaction with the electron $(\delta=3 \mathrm{meV})$, and in (c) the outer dots are effectively deeper $(\delta=-3 \mathrm{meV})$. Plot (b) well corresponds to the lowest avoided crossing of Figs. 15(b) and 15(c) to the second avoided crossing of Fig. 15(b). 
each other near $F=0$. In the second avoided crossing the first and second energy levels become closer in a similar way. In the lowest avoided crossing of Fig. 15(b) the electron stays rather in the two central dots [see Figs. 16(e)-16(h)], which makes them effectively deeper for the hole. Accordingly we take $\delta=3 \mathrm{meV}$ and obtain the features of the lowest-energy avoided crossing of Fig. 15(b). Opposite value of $\delta$, corresponding to the electron localized in the dots 1 and 4 [see Figs. 16(a)-16(d)], produces an avoided crossing with twolowest energy levels approaching each other near $F=0$, like in the second-energy multiple avoided crossing of Fig. 15(b).

\section{DISCUSSION}

Our results for intermediate coupling of $N$ identical dots show that the exciton spectrum can be divided into $N$ families, each associated with the localization of the hole in one of the dots within the stack. The energy levels of different families cross while avoided crossings are observed between bright levels of the same family as function of the external electric field which transfers the electron within the stack of $N$ dots. Each family contains $N$ energy levels that appear as bright for a certain electric field range. In each family we have a single energy level which remains bright even at strong negative electric field $F \ll 0$ and another that stays bright for $F \gg 0$. Below we refer to these energy levels as "ultimate." These two energy levels undergo an avoided crossing around $F$ close to zero. Below we call this avoided crossing as the "principle" one. For previously studied case of an exciton in two dots ${ }^{8-15}$ there are no other bright energy levels than the ultimate ones. However, for $N \geqslant 3$, the remaining $N-2$ levels of the family become bright in a finite electric field range contained within the principle avoided crossing. These temporarily bright energy levels depend on the external field in a much stronger way than the ultimate bright levels in the strong field limit. In the limit of a strong field, when both the electron and the hole are localized in the same dot, the $F$ dependence of the ultimate levels is only due to a single-dot exciton polarizability. ${ }^{35}$ On the other hand, the intrinsic and induced dipole moments of the temporarily bright energy levels are much larger, since they appear in the spectrum when the electron is removed from the dot in which the hole is localized. For the families of energy levels associated with the dots at the extreme ends of the stack all the $N-2$ temporarily bright levels depend on the electric field nearly linearly. Their slope is related to the dipole moment of the electron-hole pair when it becomes dissociated [this is most clearly visible in Fig. 14(a)]. For the families associated with the dot situated inside the stack a single temporarily bright energy level strongly deviates from the nearly linear dependence on $F$. Its dependence on the field resembles a parabola (deformed for odd $N$ ) with arms pointing down on both sides of its maximum located close to $F=0$. Position of the dot inside the stack with which the parabolic level is associated can be deduced by counting the number of the temporarily bright energy levels that are nearly linear in $F$ and enter between this parabolic energy level and the ultimate bright energy levels at $F \ll 0$ and $F \gg 0$. Namely, the number of bright linear energy levels appearing at the posi- tive field side of the maximum of the parabolic bright energy level is equal to the number of dots between the holecontaining dot (defining the family) and the extreme dot at the top of the stack, and conversely for $F<0$. Thus, addition of a $N$ th dot to a stack containing $N-1$ dots results in the appearance of a single new temporarily bright level in each of the preexisting families. This new level depends nearly linearly on the electric field. Obviously, a new family is formed with the added dot. When a dot is added to the stack the number of internal dots is increased by one and a single new temporarily bright parabolic (and not linear) energy level appears in the spectrum of the entire stack. For instance, when the third dot is added to a stack of two dots the first parabolic level appears-the one labeled by $2 b$ in Fig. 3. For $N=4$ we have already two such parabolic levels: $2 b$ and $3 c$ - see Fig. 12-which cross at $F=0$.

For the intermediate coupling the energy levels of the hole alone are $\mathrm{N}$-fold degenerate. This degeneracy is lifted by the interaction with the electron which tends to be localized in the center of the stack, where we have a single dot for odd $N$ and a couple of dots for even $N$. This provides a general rule that at $F=0$ for $N$ identical dots in the intermediate coupling the ground state is nondegenerate for odd $N$ and twofold degenerate for even $N$.

For strongly coupled dots hole tunnel coupling becomes active. According to our results, even in the extreme case of $1 \mathrm{~nm}$ thin barrier the coupling is removed by a relatively weak electric field of about $20 \mathrm{kV} / \mathrm{cm}$. When the hole coupling is removed by the electric field, the discussion of the spectrum in terms of families of energy levels associated with the hole localized in one of the dots is valid again. However, mixing of the hole states is found for small values of the field in the avoided crossing in which $N$ bright energy levels participate with a single member of each family. The participating energy levels are associated with the same single-particle state of the electron. Due to the strong electron tunnel coupling, within the range of each of these avoided crossings, the electron localization is only weakly perturbed by the electric field, and this is the hole which is redistributed between the dots by the field. This is the inverse mechanism to the one observed for the avoided crossings in the intermediate-coupling regime, where the localization of the hole is fixed and only the electron localization within the stack is changed by the electric field. The avoided crossings related to the hole tunneling and mixing between the families have two characteristic features: (1) In the center of each avoided crossing a single energy level becomes much brighter at the expense of the recombination probabilities of the other participating levels. A distinctly larger recombination probability is obtained for the hole state which is compatible with the state of the electron, i.e., corresponds to the same excitation in the direction parallel to the axis of the stack. This results in the rule that within the avoided crossing that is $m$ th in the energy scale (i.e., associated with the $m$ th excitation of the electron) it is the $m$ th of the participating energy levels which becomes the brightest in the center of the avoided crossings. (2) The order of the bright energy levels as they reappear outside the avoided crossing depends only on the position of the specific dot within the stack in which the hole becomes trapped. On the positive $F$ side of 
the avoided crossing, lower energies correspond to the hole localization in the lower dots $[F>0$ pushes the hole downsee Fig. 1(a)]. The avoided crossings that are due to the hole tunneling - with the spectacular modulation of the recombination probabilities - are only deformed by the confinement variation. The order of the subsequent avoided crossings in energy remains unchanged by the dot variation since their character is defined by the state of the electron and the electron energy splitting is huge when the dots are close enough for the hole to tunnel.

In the intermediate-coupling regime the variation of the depths of the dot within the stack leads to energy shifts of the families of energy levels. The families are also translated on the electric field scale. Nevertheless, the pattern of the avoided crossing remains characteristic to the position of the hole-containing dot within the stack. In the strong-coupling regime the variation of the confinement depths within the stack of the order of $10 \mathrm{meV}$ is much smaller than the coupling-related splitting of electron energy levels. Then, the difference of the confinement depths may not be resolved by the electron so the spectra of the strongly coupled dots are less sensitive to the confinement variation along the stack [cf. Fig. 10 for strongly coupled triple of identical dots (a) or nonidentical dots (c)].

\section{SUMMARY AND CONCLUSIONS}

We calculated the exciton spectra in triple and quadruple vertically stacked self-assembled quantum dots in the presence of an external electric field using the configurationinteraction approach. Intermediate- and strong-coupling regimes were considered and we explained how the spectra evolve when an additional dot is added to the stack.

In the intermediate-coupling regime the bright energy levels can be separated into families each associated with a specific dot of the stack in which the hole is localized. The electron transfer between the dots induced by the electric field is associated with the appearance of a pattern of avoided crossings that is characteristic for each family of energy levels. The structure of the avoided crossing is related mainly to the position of the dot within the stack which is translated into the number of avoided crossings observed for opposite electric field orientations. We found that the pattern of avoided crossing is related to the electron transfer between the dots which remains qualitatively similar when the depths of the dots are varied. The depth variation results in relative shifts of the families of energy levels. Therefore, photoluminescence experiment should be able to probe the variation of the depth of the effective confinement potential along the stack. In particular, the experimental observation of the pattern of avoided crossings for the lowest bright energy levels should indicate which of dots is the deepest within the stack.

We discussed the avoided crossings between levels of different families that appear for strongly coupled dots. These anticrossings appear at small $F$ and involve $N$ bright energy levels, where $N$ is the number of the dots within the stack. Within each anticrossing one of the energy levels increases in brightness at the expense of $N-1$ others. In the brightest state the hole occupies a single-particle orbital which is compatible with the orbital of the electron, i.e., corresponds to the same single-particle state. The mixing between families occurs only for relatively weak electric fields. For stronger fields the hole is localized in a single dot and the pattern of avoided crossings becomes qualitatively similar to the intermediate-coupling case.

\section{ACKNOWLEDGMENTS}

This work was supported by the EU Network of Excellence: SANDiE and the Belgian Science Policy (IAP).
${ }^{1}$ D. Loss and D. P. DiVincenzo, Phys. Rev. A 57, 120 (1998).

${ }^{2}$ F. Troiani, U. Hohenester, and E. Molinari, Phys. Rev. B 62, R2263 (2000).

${ }^{3}$ G. S. Solomon, J. A. Trezza, A. F. Marshall, and J. S. Harris, Jr., Phys. Rev. Lett. 76, 952 (1996).

${ }^{4}$ N. N. Ledentsov, V. A. Shchukin, M. Grundmann, N. Kirstaedter, J. Bohrer, O. Schmidt, D. Bimberg, V. M. Ustinov, A. Yu. Egorov, A. E. Zhukov, P. S. Kop'ev, S. V. Zaitsev, N. Yu. Gordeev, Zh. I. Alferov, A. I. Borovkov, A. O. Kosogov, S. S. Ruvimov, P. Werner, U. Gosele, and J. Heydenreich, Phys. Rev. B 54, 8743 (1996).

${ }^{5}$ O. G. Schmidt, O. Kienzle, Y. Hao, K. Eberl, and F. Ersnt, Appl. Phys. Lett. 74, 1272 (1999).

${ }^{6}$ B. Lita B, R. S. Goldman, J. D. Phillips, and P. K. Bhattacharya, Appl. Phys. Lett. 74, 2824 (1999).

${ }^{7}$ S. Fafard, M. Spanner, J. P. McCaffrey, and Z. R. Wasilewski, Appl. Phys. Lett. 76, 2268 (2000).

${ }^{8}$ H. J. Krenner, M. Sabathil, E. C. Clark, A. Kress, D. Schuh, M. Bichler, G. Abstreiter, and J. J. Finley, Phys. Rev. Lett. 94, 057402 (2005).
${ }^{9}$ E. A. Stinaff, M. Scheibner, A. S. Bracker, I. V. Pomonarev, V. L. Korenev, M. E. Ware, M. F. Doty, T. L. Reinecke, and D. Gammon, Science 311, 636 (2005).

${ }^{10}$ G. Ortner, M. Bayer, Y. Lyanda-Geller, T. L. Reinecke, A. Kress, J. P. Reithmaier, and A. Forchel, Phys. Rev. Lett. 94, 157401 (2005).

${ }^{11}$ A. S. Bracker, M. Scheibner, M. F. Doty, E. A. Stinaff, I. V. Pomonarev, J. C. Kim, L. J. Whitman, T. L. Reinecke, and D. Gammon, Appl. Phys. Lett. 89, 233110 (2006).

${ }^{12}$ H. J. Krenner, E. C. Clark, T. Nakaoka, M. Bichler, C. Scheurer, G. Abstreiter, and J. J. Finley, Phys. Rev. Lett. 97, 076403 (2006).

${ }^{13}$ T. Nakaoka, E. C. Clark, H. J. Krenner, M. Sabathil, M. Bichler, Y. Arakawa, G. Abstreiter, and J. J. Finley, Phys. Rev. B 74, 121305(R) (2006).

${ }^{14}$ M. F. Doty, M. Scheibner, I. V. Ponomarev, E. A. Stinaff, A. S. Bracker, V. L. Korenev, T. L. Reinecke, and D. Gammon, Phys. Rev. Lett. 97, 197202 (2006).

${ }^{15}$ M. Scheibner, M. F. Doty, I. V. Ponomarev, A. S. Bracker, E. A. Stinaff, V. L. Korenev, T. L. Reinecke, and D. Gammon, Phys. 
Rev. B 75, 245318 (2007).

${ }^{16}$ E. Biolatti, R. C. Iotti, P. Zanardi, and F. Rossi, Phys. Rev. Lett. 85, 5647 (2000); P. Chen, C. Piermarocchi, and L. J. Sham, ibid. 87, 067401 (2001).

${ }^{17}$ M. W. Keller, J. M. Martinis, N. M. Zimmerman, and A. H. Steinbach, Appl. Phys. Lett. 69, 1804 (1996).

${ }^{18}$ M. Klein, S. Rogge, F. Remacle, and R. D. Levine, Nano Lett. 7, 2795 (2007).

${ }^{19}$ D. S. Saraga and D. Loss, Phys. Rev. Lett. 90, 166803 (2003).

${ }^{20}$ L. Gaudreau, S. A. Studenikin, A. S. Sachrajda, P. Zawadzki, A. Kam, J. Lapointe, M. Korkusinski, and P. Hawrylak, Phys. Rev. Lett. 97, 036807 (2006); D. S. Saraga and D. Loss, ibid. 90, 166803 (2003).

${ }^{21}$ M. Schmidbauer, S. Seydmohamadi, D. Grigoriev, Z. M. Wang, Yu. I. Mazur, P. Schafer, M. Hanke, R. Kohler, and G. J. Salamo, Phys. Rev. Lett. 96, 066108 (2006).

${ }^{22}$ J. L. Gray, R. Hull, and J. A. Fioro, J. Appl. Phys. 100, 084312 (2006).

${ }^{23}$ J. H. Lee, Zh. M. Wang, N. W. Strom, Yu. I. Mazur, and G. J. Salamo, Appl. Phys. Lett. 89, 202101 (2006).

${ }^{24}$ B. Szafran, T. Chwiej, F. M. Peeters, S. Bednarek, J. Adamowski, and B. Partoens, Phys. Rev. B 71, 205316 (2005).

${ }^{25}$ W. Chu and J. L. Zhu, Appl. Phys. Lett. 89, 053122 (2006).

${ }^{26}$ M. H. Degani, G. A. Farias, and P. F. Farinas, Appl. Phys. Lett.
89, 152109 (2006).

${ }^{27}$ B. Szafran, F. M. Peeters, and S. Bednarek, Phys. Rev. B 75, 115303 (2007)

${ }^{28}$ M. H. Degani and M. Z. Maialle, Phys. Rev. B 75, 115322 (2007).

${ }^{29}$ R. L. Sellin, Ch. Ribbat, M. Grundmann, N. N. Ledentsov, and D. Bimberg, Appl. Phys. Lett. 78, 1207 (2001).

${ }^{30}$ R. L. Sellin, C. Ribbat, D. Bimberg, F. Rinner, H. Konstanzer, M. T. Kelemen, and M. Mikulla, Electron. Lett. 38, 883 (2002).

${ }^{31}$ G. Ya. Slepyan, S. A. Maksimenko, V. P. Kalosha, J. Hermann, N. N. Ledentsov, I. L. Krestnikov, Zh. I. Alferov, and D. Bimberg, Phys. Rev. B 59, 12275 (1999); G. Ya. Slepyan, S. A. Maksimenko, V. P. Kalosha, A. Hoffmann, and D. Bimberg, ibid. 64, 125326 (2001).

${ }^{32}$ M. Scheibner, T. Schmidt, L. Worschech, A. Forchel, G. Bacher, T. Passow, and D. Hommel, Nat. Phys. 3, 106 (2007).

${ }^{33} \mathrm{~A}$ small electric field of $1 \mathrm{kV} / \mathrm{cm}$ was assumed in Figs. 4(a) and 4(b) to lift the parity symmetry of the states. In a system of symmetric dots, due to the parity symmetry, at $F=0$ the hole resides equally in all the dots in spite of the absence of interdot hole tunnelling.

${ }^{34}$ B. Szafran, S. Bednarek, and J. Adamowski, Phys. Rev. B 64, 125301 (2001).

${ }^{35}$ J. A. Barker and E. P. O’Reilly, Phys. Rev. B 61, 13840 (2000). 